\title{
Analysis of Sting Balance Calibration Data Using Optimized Regression Models
}

\author{
N. Ulbrich* \\ Jacobs Technology Inc., Moffett Field, California 94035-1000 \\ J. Bader** \\ NASA Ames Research Center, Moffett Field, Califormia 94035-1000
}

Calibration data of a wind tunnel sting balance was processed using a candidate math model search algorithm that recommends an optimized regression model for the data analysis. During the calibration the normal force and the moment at the balance moment center were selected as independent calibration variables. The sting balance itself had two moment gages. Therefore, after analyzing the connection between calibration loads and gage outputs, it was decided to choose the difference and the sum of the gage outputs as the two responses that best describe the behavior of the balance. The math model search algorithm was applied to these two responses. An optimized regression model was obtained for each response. Classical strain-gage balance load transformations and the equations of the deflection of a cantilever beam under load are used to show that the search algorithm's two optimized regression models are supported by a theoretical analysis of the relationship between the applied calibration loads and the measured gage outputs. The analysis of the sting balance calibration data set is a rare example of a situation when terms of a regression model of a balance can directly be derived from first principles of physics. In addition, it is interesting to note that the search algorithm recommended the correct regression model term combinations using only a set of statistical quality metrics that were applied to the experimental data during the algorithm's term selection process.

\section{Nomenclature}

$a$
$b$
$c_{1}$
$c_{2}$
$d$
$d_{1}$
$d_{2}$
$d_{*}$
$E$
$F$
$F_{1}$
$F_{2}$
$h$
$I$
$k_{1}$ $=$ lower integration interval bound

$=$ upper integration interval bound

$=$ coordinate of the center of the forward balance gage

$=$ coordinate of the center of the aft balance gage

$=$ distance between the center of the forward and the center of the aft balance gage

$=$ first auxiliary coordinate difference

$=$ second auxiliary coordinate difference

$=$ distance between model moment center and balance moment center

$=$ modulus of elasticity

$=$ force at the model moment center and at the balance moment center

$=$ force at the center of the forward balance gage

$=$ force at the center of the aft balance gage

$=$ distance between balance calibration fixture and balance reference axis

$=$ area moment of inertia

$=$ first regression coefficient of deflection angle equation at free end of sting

* Aerodynamicist, Jacobs Technology Inc.

** Branch Chief, Code AOI, NASA Ames Research Center

Copyright $\odot \mathbf{2 0 0 9}$ by the American Institute of Aeronautics and Astronautics, Inc. - The U.S. Government has a royalty-free license to exercise all rights under the copyright claimed herein for Governmental purposes. All other rights are reserved by the copyright owner. 


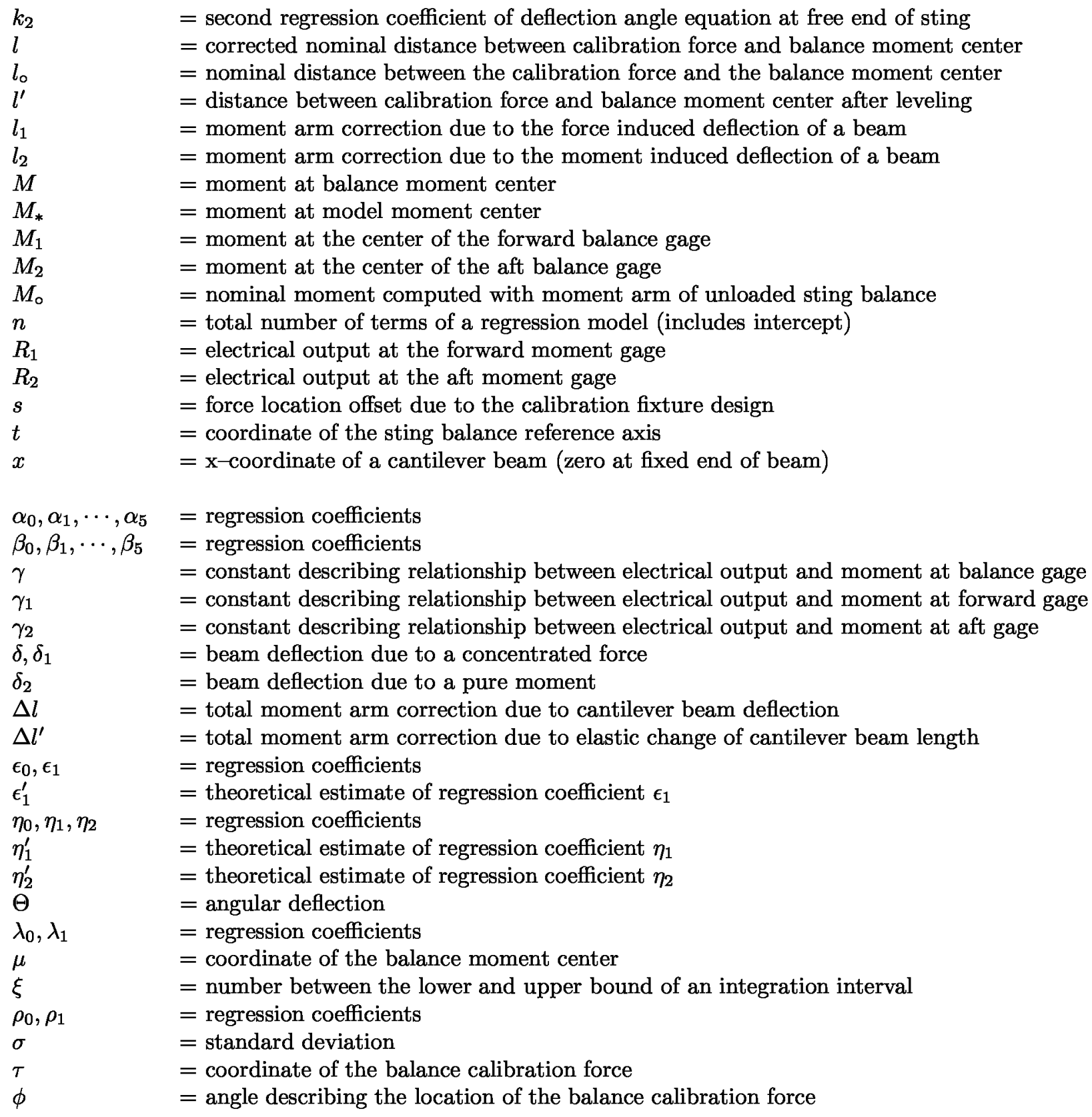

\section{Introduction}

During the past 4 years a software package called BALFIT was developed for the Wind Tunnel Division at NASA Ames Research Center that is used for the regression analysis of wind tunnel strain-gage balance calibration data. The software uses an innovative candidate math model search algorithm in order to find an optimized regression model for the analysis of data. The algorithm is not limited to wind tunnel balance calibration data applications. It can also be applied to more general multivariate experimental data sets.

In principle, the search algorithm tries to identify an optimized regression model, i.e., the so-called recommended math model, that has superior predictive capabilities. It is obtained after minimizing a search metric that is a measure of the regression model's expected predictive capability. In addition, two constraints are applied that make it possible to test only those regression models during the search that meet strict statistical quality requirements. A detailed description of the search algorithm's approach can 
be found in Refs. [1], [2], and [3].

So far, the search algorithm has been applied to a wide variety of wind tunnel balance calibration data sets. Traditional hand load data sets of multi-piece, single-piece, and floor balances were processed successfully (Refs. [4], [5]). In addition, large data sets from calibration machines were also analyzed (Refs. [6], [7]). A calibration data set of a wind tunnel sting balance was analyzed for the first time in 2008. This data set was courteously supplied to the authors by The Gulfstream Aerospace Corporation. During the analysis of the sting balance data set an interesting connection between the optimized regression model of the data and a first principles analysis of the relationship between the calibration loads and the gage outputs was observed. This connection is discussed in great detail in the present paper. At first, however, basic assumptions and elements of the regression model optimization algorithm are reviewed in order to provide a better understanding the regression model optimization process.

\section{Regression Model Optimization Algorithm}

Figure 1 shows basic elements of the most recent version of the candidate math model search algorithm. The goal of the algorithm is to find an optimized regression model, i.e., the so-called recommended math model, that best represents the given experimental data set. The regression model search (optimization) is performed in several steps. Initially, the user of the algorithm has to select a function class combination that appears to be most suitable for the global regression analysis of the experimental data. In the next step, the upper bound for the regression model search has to be identified. This upper bound is called the "permitted math model." It is the largest possible math model for the given function class combination and data set that will lead to a non-singular solution of the global regression problem. This upper bound makes it possible to perform an automated regression model search as it is the largest regression model in the given context that will not lead to a software "crash" during the regression model search.

How can the upper bound of the math model search space be identified? This question is answered in some detail in Ref. [3]. The basic approach of the identification process can be summarized in a few sentences. First, each math term of the regression model of the responses is considered to be a column vector that has a number of rows equal to the number of data points of the data set. A non-singular solution of the global regression problem can only exist if the column vectors representing the math terms are linearly independent. They have to be basis vectors of a vector space.

At this point another question emerges. How can the linear independence of a set of vectors be tested? A numerical technique called Singular Value Decomposition (SVD) has to be applied "iteratively" for this purpose (see also the discussion in Ref. [3], p.5). What does "iteratively" mean in this context? Let us assume, for example, that a regression model of the responses consist of a total number of " $n$ " terms (including the intercept term). Then, a total number of " $n$ " column vectors can be defined that represent all possible math terms of a regression model of the data set for the given function class combination. Now, the first vector, e.g., the vector of ones representing the intercept term, is considered to be the initial basis vector of a vector space. In the next step, SVD is used to test if the second vector is linearly independent of the first vector. Only two possibilities exist. Possibility 1: The second vector is linearly independent of the first vector $\Longrightarrow$ a new vector space is defined using the first and second vector $\Longrightarrow$ the new vector space will be used to test the third vector. Possibility 2: The second vector depends linearly on the first vector $\Longrightarrow$ the second vector is no longer considered to be a new basis vector of a vector space $\Longrightarrow$ the corresponding math term is removed from the regression model $\Longrightarrow$ the original vector space remains unchanged and will be used to test the third vector. The process is repeated for each vector. The vector space is increased, of course, by using the tested vector whenever this vector is linearly independent of the vector space that is defined by the previously found linearly independent set of vectors. Consequently, SVD has to be applied a total number of " $n-1$ " times in order to identify and remove all potentially linearly dependent vectors (math terms) that may be contained in the largest theoretically possible regression model. The remaining set of vectors (math terms) defines the final vector space for the given data set and function class combination that will always lead to a non-singular solution of the global regression problem.

Theoretically, no order of the column vectors (math terms) has to be specified in order to test column vectors for linear dependency using the suggested iterative application of SVD. Lower order terms of the regression model may be tested before -or- after higher order terms. In a practical implementation, however, 
testing lower order terms before higher order terms may provide a better understanding of the reason why a certain column vector (math term) was rejected during the iterative application of SVD.

The search for the optimized regression model can begin after the upper bound of the math model search space has been identified by applying SVD to the selected function classes and data set. The search starts at the lower bound of the search space which, for wind tunnel strain-gage balance calibration data applications, is defined by the intercept and the principle linear load term that is connected with a specific balance gage output. For a sting balance, for example, the principle linear load term for the difference of the gage outputs is the normal force at the balance moment center (BMC). A metric, i.e., the standard deviation of the PRESS residuals of the responses is minimized during the search. This metric is used to compare the predictive capability of different regression models that are tested during the search (see also the flowchart in Fig. 1). A primary and secondary constraint are enforced during the search. The primary search constraint makes sure that a tested regression model only has statistically significant terms. The secondary search constraint helps avoid math models that have unwanted near-linear dependencies. In the end, only those regression models are considered during the search that fulfill both constraints (see Ref. [1] for more details about the search metric and the application of the two search constraints).

The user has the option to apply the "hierarchy rule" to the optimized regression model after the completion of the search. The "hierarchy rule" adds missing lower order terms to a regression model. The enforcement of the "hierarchy rule" is "optional" as its application will not necessarily lead to a regression model with better predictive capabilities (a detailed discussion of this viewpoint is given in Ref. [1]). Previously, the search algorithm also allowed the user to enforce the "hierarchy rule" during the candidate math model search. This option is no longer supported as it appears to lead to suboptimal search results for certain types of data sets.

In the next part of the paper the sting balance's design and its calibration are discussed in more detail. Then, results of the regression model optimization of the calibration data will be presented.

\section{Wind Tunnel Sting Balance Design and Calibration}

The selected sting balance has two moment gages that are mounted forward and aft of the BMC. The drawing in Fig. 2 shows the location of the two moment gages relative to the BMC. The balance has an approximate total length of 28 [in] and a $30^{\circ}$ bend angle at the midpoint. Therefore, the centerline of the test article is about 5 [in] below the centerline of the downstream portion of the balance.

Figure 3a shows a sideview of the sting balance as it appeared during the calibration. The balance gages are covered using gage fairings. In addition, an Angle Measurement System (AMS) unit is attached to the test article mounting plate. The AMS unit was used to level the test article mounting plate during calibration. Figure $3 \mathrm{~b}$ shows a more detailed view of the forward and aft gage after the removal of the gage fairings.

The calibration of the sting balance was performed using weights that were applied in both normal and upside-down orientation of the balance. Figure 3c shows the balance, AMS unit, the calibration fixtures, and weights in upside-down orientation. This orientation was used to apply a positive normal force during the calibration. Four horizontal load points were used on the calibration equipment in order to vary the distance between the normal force and the BMC. Figure 4 shows all force and moment combinations that were applied during the calibration of the balance. Four distinct lines can be seen in Fig. 4. They are related to the four moment arms that were chosen for the calibration (16.52 [in], 18.52 [in], 20.52 [in], 25.92 [in]).

The forward balance gage measures a raw electrical output called $R_{1}$. The aft gage measures a raw electical output called $R_{2}$. Both gages are moment gages. Consequently, the gage output $R_{1}$ is approximately proportional to the moment $M_{1}$ at the forward gage and the gage output $R_{2}$ is approximately proportional to the moment $M_{2}$ at the aft gage. Two principle combinations of regressors and responses are possible in order to perform a regression analysis of the calibration data. The two options depend on the balance load format that is selected for the regression analysis. The moments $M_{1}$ and $M_{2}$ at the forward and aft gage may be used as independent variables for the regression analysis. In that case, the electrical outputs $R_{1}$ and $R_{2}$ are good choices for the responses. However, it is also possible to select the force $F$ and moment $M$ at the $\mathrm{BMC}$ as independent variables. Then, the linear combinations $R_{2}-R_{1}$ and $R_{1}+R_{2}$ should be selected for the analysis as $R_{2}-R_{1}$ is nearly proportional to $F$ and $R_{1}+R_{2}$ is nearly proportional to $M$. The two 
options are summarized in Table 1 below.

Table 1: Regressor and response combination options for sting balance calibration analysis.

\begin{tabular}{|l|c|c|}
\hline & REGRESSORS & RESPONSES \\
\hline \hline OPTION 1 & $M_{1}, M_{2}$ & $R_{1}, R_{2}$ \\
\hline OPTION 2 & $F, M$ & $R_{2}-R_{1}, R_{1}+R_{2}$ \\
\hline
\end{tabular}

Why is the difference $R_{2}-R 1$ nearly proportional to $F$ at the BMC? Why is the sum $R_{1}+R_{2}$ of the gage outputs nearly proportional to $M$ at the BMC? These assertions will be explained more rigorously in a later section of the paper. The advantage can also be understood if the two proposed linear combinations of the gage outputs are plotted versus the loads at the BMC. Figure 5a shows the difference of the gage outputs plotted versus the applied normal force. Figure 5b shows the sum of the gage outputs plotted versus the applied moment. In both cases we see that the selected responses are nearly proportional to the corresponding load that is applied. Therefore, any regression model developed for the difference and the sum of the gage outputs will be dominated by a single linear term if the force and moment at the BMC are used as independent variables. This characteristic greatly simplifies the development of a regression model of the calibration data. - In the next section of the paper the regression analysis of the difference and the sum of the balance gage outputs will be discussed in more detail.

\section{Regression Analysis of Calibration Data}

\section{A. General Remarks}

The selection of suitable regressors and responses of the sting balance calibration data set has a significant influence on the overall quality of the regression analysis of the data. It was decided to use the applied force $F$ and moment $M$ at the BMC as independent variables for the regression analysis. In that case, as explained above, (i) the difference $R_{2}-R_{1}$ and (ii) the sum $R_{1}+R_{2}$ of the moment gage outputs should be used as responses. Two different regression model types were selected for each response in order to demonstrate the benefit of using an optimized regression model for the analysis of the balance calibration data. The first regression model type is a traditional quadratic. The second regression model type is the optimized regression model that the candidate search algorithm selected. At first, the regression analysis results for the traditional quadratic are discussed. Afterwards, these results will be compared with corresponding results that were obtained using the optimized regression models of the calibration data.

\section{B. Traditional Quadratic}

A traditional quadratic may be selected for the regression analysis of the difference of the gage outputs. Then, the difference may be modeled in the least squares sense using the following math model:

$$
\text { Quadratic 1: } R_{2}-R_{1}=\alpha_{0}+\alpha_{1} \cdot F+\alpha_{2} \cdot M+\alpha_{3} \cdot F^{2}+\alpha_{4} \cdot M^{2}+\alpha_{5} \cdot F \cdot M \quad(1 a)
$$

Similarly, the sum of the gage outputs may be modeled using the following quadratic:

$$
\text { Quadratic 2: } \quad R_{1}+R_{2}=\beta_{0}+\beta_{1} \cdot F+\beta_{2} \cdot M+\beta_{3} \cdot F^{2}+\beta_{4} \cdot M^{2}+\beta_{5} \cdot F \cdot M
$$

Figure 6a shows regression analysis results for the difference of the gage outputs if the traditional quadratic is used for the analysis. This math model has variance inflation factors (VIFs) that are significantly larger that the literature recommended liberal threshold of 10 (see list of VIFs printed in red in Fig. 6a). The math model described in Eq. (1a) has massive near-linear dependencies. Therefore, its predictive capability 
is put into question even though the standard deviation of the fitted residuals of the responses is only $0.0836 \%$ of the largest response magnitude.

Figure $6 \mathrm{~b}$ shows corresponding regression analysis results for the sum of the gage outputs if a traditional quadratic is used for the analysis. Again, the VIFs are large indicating the presence of massive near-linear dependencies. Consequently, the predictive capability of the regression model given in Eq. (1b) is also put into question even though the standard deviation of the fitted residuals of the responses is only $0.0393 \%$ of the largest response magnitude.

\section{Optimized Regression Model}

In the next phase of the data analysis the regression model optimization algorithm defined in Fig. 1 was applied to the difference and the sum of the gage outputs. First, a lower and an upper bound for the candidate math model search had to be defined. It was decided to use a simple linear math model as the lower bound and a traditional quadratic as the upper bound for the candidate math model search. Then, we get for the difference of the gage outputs the following bounds:

$$
R_{2}-R_{1}=\left\{\begin{array}{l}
\text { Lower Bound 1: } \rho_{0}+\rho_{1} \cdot F \\
\text { Upper Bound 1: } \alpha_{0}+\alpha_{1} \cdot F+\alpha_{2} \cdot M+\alpha_{3} \cdot F^{2}+\alpha_{4} \cdot M^{2}+\alpha_{5} \cdot F \cdot M
\end{array}\right.
$$

The search was performed using the most conservative thresholds for both the primary and secondary search constraint: (1) $p$-value of the $t$-statistic threshold $\Longrightarrow 0.0001$ and (2) VIF threshold $\Longrightarrow 5$. A total number of 20 regression models were tested during the search. The optimized regression model, i.e., the recommended math model, was obtained after the completion of the search. It has the following form:

$$
\text { Optimized Model 1: } \quad R_{2}-R_{1}=\epsilon_{0}+\epsilon_{1} \cdot F
$$

Figure 7a shows the regression analysis results for the difference of the gage outputs if the optimized regression model defined in Eq. (2c) is used for the analysis. The regression model has no near-linear dependencies as the VIFs are significantly smaller that the liberal threshold of 10 . In addition, only two terms are used (instead of six for the traditional quadratic). The standard deviation of the fitted residuals of the difference is only $0.1056 \%$ of the largest response magnitude. This value is only slightly larger than the value that was obtained for the traditional quadratic (see Fig. 6a). Therefore, it can be concluded that the traditional quadratic, i.e., Eq. (1a), significantly overfitted the difference of the gage outputs. Overfitting of experimental data should be avoided as it can greatly reduce the predictive capability of a regression model at data points that were not used for the regression analysis.

The regression model optimization was applied to the sum of the gage outputs in a similar fasion. Again, the lower and upper bound for the candidate math model search were selected to be:

$$
R_{1}+R_{2}=\left\{\begin{array}{l}
\text { Lower Bound 2: } \lambda_{0}+\lambda_{1} \cdot M \\
\text { Upper Bound 2: } \beta_{0}+\beta_{1} \cdot F+\beta_{2} \cdot M+\beta_{3} \cdot F^{2}+\beta_{4} \cdot M^{2}+\beta_{5} \cdot F \cdot M
\end{array}\right.
$$

In the next phase of the analysis the candidate math model search algorithm was applied to the sum of the gage outputs. In that case, the algorithm chose the following recommended math model:

$$
\text { Optimized Model 2: } \quad R_{1}+R_{2}=\eta_{0}+\eta_{1} \cdot M+\eta_{2} \cdot M^{2}
$$

Figure 7b shows regression analysis results for the sum of the gage outputs if the optimized regression model defined in Eq. (3c) is used for the analysis. The regression model has no near-linear dependencies as the VIFs are significantly smaller that the liberal threshold of 10 . In addition, only three terms are used 
(instead of six for the traditional quadratic). The standard deviation of the fitted residuals of the difference is only $0.0472 \%$ of the largest response magnitude. This value is only slightly larger than the value that was obtained for the traditional quadratic (see Fig. 6b). Again, it can be concluded that the traditional quadratic, i.e., Eq. (1b), significantly overfitted the sum of the gage outputs.

It is a surprising result of the regression model search that (i) the optimized model of the difference of the gage outputs only depends on the intercept and the normal force and that (ii) the optimized model of the sum of the gage outputs is only a function of the intercept, the moment, and the square of the moment. An explanation of these search results had to be found.

Fortunately, the overall geometry and design of the sting balance is very simple. Therefore, it will be shown analytically in the next section of the paper that the optimized regression model of the difference of the gage outputs (i.e., Eq. (2c)) can be obtained after applying classical strain-gage balance load transformations to the sting balance.

It is suspected that the term selection of the optimized regression model of the sum of the gage outputs (i.e., Eq. (3c)) is primarily influenced by the elastic deformation characteristics of the sting balance. Therefore, it was decided to use the equations of the deflection of a cantilever beam in order to develop an expression for the connection between the balance loads at the BMC and the sum of the gage outputs that can be compared with the corresponding optimized regression model. Results of this second theoretical analysis will also be presented in one of the next sections of the paper.

\section{Theoretical Regression Model of the Difference of the Gage Outputs}

In this section it is shown how the optimized regression model of the difference of the sting balance gage outputs (Eq. (2c)) can be obtained if the classical strain-gage balance load transformation equations are applied to the sting balance. At first, an analysis of the connection between the balance loads needs to be performed. It is concluded from Fig. 8 that the moment at the BMC is given by the following expression:

$$
M=F \cdot[\mu-\tau]
$$

where $F$ is the normal force that is applied in the vicinity of the free end of the sting balance. The sting balance has moment gages, i.e., the gage outputs are proportional to moments at the gage location. Therefore, in order to develop the relationship between the gage outputs and the force and moment at the BMC, the moment at the forward and aft gage needs to be expressed as a function of the loads at the BMC. Using again Fig. 8, the moments $M_{1}$ and $M_{2}$ at the center of the forward and aft gage may be written as:

$$
\begin{aligned}
& M_{1}=F \cdot\left[\mu-\tau-\frac{d}{2}\right]=F \cdot[\mu-\tau]-\frac{F}{2} \cdot d \\
& M_{2}=F \cdot\left[\mu-\tau+\frac{d}{2}\right]=F \cdot[\mu-\tau]+\frac{F}{2} \cdot d
\end{aligned}
$$

Then, after inserting Eq. (4) into Eqs. (5a) and (5b), we get:

$$
\begin{aligned}
& M_{1}=M-\frac{F}{2} \cdot d \\
& M_{2}=M+\frac{F}{2} \cdot d
\end{aligned}
$$

Equations $(6 a)$ and $(6 b)$ are the classical strain-gage balance load transformations for a moment type balance assuming that the BMC is located halfway between the forward and aft gage (see enclosed appendix for a completed derivation of the classical strain-gage balance load transformations). Equations (6a) and $(6 b)$ are also a linear system of two equations in two unknowns that may be used to obtain the relationship between the loads $F$ and $M$ at the BMC and the moments $M_{1}$ and $M_{2}$ at the electrical centers of the gages. After some algebra, we get the solution of this linear system:

$$
F=\frac{M_{2}-M_{1}}{d}
$$




$$
M=\frac{M_{1}+M_{2}}{2}
$$

As expected, Eqs. (7) and (8) agree with Eqs. $(A .24 a)$ and $(A .24 b)$ for a moment balance that are derived in great detail in the appendix of the present paper. Now, the relationship between the loads at the BMC and the moments at the gages is known. It remains to find the relationship between (i) the electrical outputs and (ii) the moments at the gages. As mentioned above, it is simply assumed that the gage outputs are proportional to the moments at the electrical center of the gages. Then, we get the following relationships:

$$
\begin{aligned}
& R_{1}=\gamma_{1} \cdot M_{1} \\
& R_{2}=\gamma_{2} \cdot M_{2}
\end{aligned}
$$

The sting balance calibration data set may be used to get the numerical values for the two constants of proportionality. A least squares analysis of the calibration data leads to the following results:

$$
\begin{aligned}
& \gamma_{1}\left(R_{1}, M_{1}\right) \approx 2.1643\left[\frac{\mu V / V}{i n-l b s}\right] \quad \text { where } \sigma=0.0046\left[\frac{\mu V / V}{i n-l b s}\right] \\
& \gamma_{2}\left(R_{2}, M_{2}\right) \approx 2.1774\left[\frac{\mu V / V}{i n-l b s}\right] \quad \text { where } \sigma=0.0044\left[\frac{\mu V / V}{i n-l b s}\right]
\end{aligned}
$$

Comparing the right hand sides of Eqs. $(10 a)$ and $(10 b)$ we see that the first derivatives of the two gage outputs are almost identical. Therefore, the following assumption can be made:

$$
\gamma=\gamma_{1}=\gamma_{2}
$$

The constant $\gamma$ may be computed by fitting a line to the combined calibration data set. We get:

$$
\gamma\left(R_{1}, R_{2}, M_{1}, M_{2}\right) \approx 2.1709\left[\frac{\mu V / V}{i n-l b s}\right] \quad \text { where } \quad \sigma=0.0079\left[\frac{\mu V / V}{i n-l b s}\right]
$$

Consequently, the final relationship between the electrical output and the moments at the two gages becomes:

$$
\begin{aligned}
& R_{1}=\gamma \cdot M_{1} \\
& R_{2}=\gamma \cdot M_{2}
\end{aligned}
$$

Now, the difference between the two gage outputs can be computed by subtracting the right hand side of Eq. (12a) from the right hand side of Eq. (12b). Then, we get:

$$
R_{2}-R_{1}=\gamma \cdot\left[M_{2}-M_{1}\right]
$$

The difference of the moments on the right hand side of Eq. (13a) still needs to be replaced. Rearranging terms in Eq. (7) we know:

$$
M_{2}-M_{1}=F \cdot d
$$

Finally, after replacing the difference of the moments in Eq. (13a) with the right hand side of Eq. (13b), we get the relationship between (i) the difference of the gage outputs and (ii) the loads at the BMC:

$$
\begin{aligned}
R_{2}-R_{1} & =\epsilon_{1}^{\prime} \cdot F \\
\epsilon_{1}^{\prime} & =\gamma \cdot d
\end{aligned}
$$


Now, the right hand side of Eq. (14a) may be compared with the right hand side of Eq. (2c). In both equations the applied calibration force $F$ is the first regressor of the sting balance calibration data set. It is important to remember that Eq. (14a) was derived assuming that the electrical outputs of the two gages do not have a systematic error. Therefore, the intercept term in the theoretical math model, i.e., Eq. (14a), is zero. The optimized regression model, i.e., Eq. (2c), was obtained using the actual data set. Therefore, the intercept term in the optimized regression model is non-zero as it picked up any systematic error that was present in the measured gage outputs.

\section{Theoretical Regression Model of the Sum of the Gage Outputs}

\section{A. Exact Regression Model}

The equations of the deflection of a cantilever beam under load may be used to show that the optimized regression model of the sum of the gage outputs, i.e., Eqs. (3c), can also be derived from first principles of engineering mechanics. At first, the sum of the gage outputs needs to be computed. Adding the right hand sides of Eq. (12a) and Eq. (12b), we get:

$$
R_{1}+R_{2}=\gamma \cdot\left[M_{1}+M_{2}\right]
$$

The sum of the moments on the right hand side of Eq. (15a) still needs to be replaced. Rearranging terms in Eq. (8) we know:

$$
M_{1}+M_{2}=2 \cdot M
$$

Now, after replacing the sum of the moments in Eq. (15a) by using the right hand side of Eq. (15b), we get the relationship between the sum of the gage outputs and the loads at the BMC:

$$
R_{1}+R_{2}=[\gamma \cdot 2] \cdot M
$$

The moment at the BMC equals the normal force times the moment arm. Therefore, the moment at the BMC can be expressed as follows:

$$
M=F \cdot l
$$

The moment arm $l$ is the true moment arm of the normal force relative to the BMC assuming that the sting balance experiences an elastic deformation. This moment arm can be expressed as the sum of (i) a "nominal" moment arm $l_{\text {o }}$ at zero force (equals the moment arm for a rigid sting) and (ii) a moment arm correction $\Delta l$ that takes the deformation of the sting balance under load into account. Then, we get:

$$
l=l_{\circ}+\Delta l
$$

Inserting the right hand side of Eq. (17) into Eq. (16), we get:

$$
M=F \cdot\left[l_{\circ}+\Delta l\right]
$$

Now, the moment $M$ in Eq. (15c) is replaced using the right hand side of Eq. (18). Then, Eq. (15c) becomes:

$$
R_{1}+R_{2}=[\gamma \cdot 2] \cdot F \cdot\left[l_{\circ}+\Delta l\right]=[\gamma \cdot 2] \cdot F \cdot l_{\circ}+[\gamma \cdot 2] \cdot F \cdot \Delta l
$$

During the balance calibration the "nominal" moment at the BMC was determined by computing the product of the applied calibration force and the "nominal" moment arm. The "nominal" moment is the second regressor of the calibration data set. It can be expressed as follows:

$$
M_{\circ}=F \cdot l_{\circ}
$$

Now, after using Eq. (20) in order to introduce the "nominal" moment in Eq. (19), we get: 


$$
R_{1}+R_{2}=[\gamma \cdot 2] \cdot M_{\circ}+[\gamma \cdot 2] \cdot F \cdot \Delta l
$$

The change of the moment arm $\Delta l$ due to the elastic deflection of the sting balance under load still needs to be determined. It is assumed that the moment arm change is primarily caused by the bending of the sting balance under load. The sting balance itself may be considered as a cantilever beam in order to quantitatively assess the moment arm change due to bending. The exact solution of this moment arm correction due to sting deformation is derived in the next section of the paper.

\section{B. Exact Solution of Moment Arm Correction}

Figure 8 shows the loads that act on the sting balance during calibration. A calibration fixture is attached to the free end of the sting. The fixture has two tasks: (i) it allows for the application of the normal force at a desired "nominal" moment arm and (ii) it makes it possible to attach an AMS unit to the balance that is needed for the leveling of the calibration fixture after the load is applied. It is important to remember that the calibration force is applied at locations on the calibration fixture that do not necessarily coincide with the free end of the sting (green dot in Fig. 8 marks the location of the calibration force on the calibration fixture). Therefore, in the general case, two types of beam bending need to be superimposed in order to estimate the moment arm change. The first bending is caused by a concentrated force (see Fig. 9a). The second bending is caused by a moment at the free end of the sting (see Fig. 9b). Consequently, the total moment arm correction is the sum of the superimposed corrections due to the two types of bending. We get:

$$
\Delta l=l_{1}+l_{2}
$$

It remains to find a relationship between the beam deflections $\delta_{1}$ and $\delta_{2}$ and the moment arm corrections $l_{1}$ and $l_{2}$. Similar triangles in Fig. 9a and Fig. $9 \mathrm{~b}$ may be used for this purpose. We get:

$$
\frac{l_{1}}{\delta_{1}}=\frac{l_{2}}{\delta_{2}}=\frac{h}{l_{\circ} / \cos \phi}
$$

Now, after solving Eq. (23) for the moment arm correction $l_{1}$, we get:

$$
l_{1}=\frac{h}{l_{\circ} / \cos \phi} \cdot \delta_{1}
$$

It is suspected that the deflection $\delta_{1}$ due to the concentrated force is significantly larger than the deflection $\delta_{2}$ due to the moment at the free end. The equations of maximum cantilever beam deflection given on p.170 of Ref. [8] may be used to investigate this hypothesis. We know, using these equations, that the ratio between $\delta_{1}$ and $\delta_{2}$ may be approximated as follows:

$$
\frac{\delta_{1}}{\delta_{2}} \approx \underbrace{\left[\frac{[F \cdot \cos \phi] \cdot\left[l_{\circ} / \cos \phi\right]^{3}}{3 \cdot E \cdot I}\right]}_{\text {concentrated force }} \cdot \underbrace{\left[\frac{2 \cdot E \cdot I}{[s \cdot F \cdot \sin \phi] \cdot\left[l_{\circ} / \cos \phi\right]^{2}}\right]}_{\text {moment at free end }} \approx \frac{2}{3} \cdot \frac{l_{\circ}}{s \cdot \sin \phi}
$$

The estimated bend angle $\phi$ of the sting in Fig. 9a and Fig. 9b is between $15^{\circ}$ and $20^{\circ}$. The moment arm $l_{\circ}$ is on the order of $20[i n]$. In addition, the distance $s$ in Fig. $9 \mathrm{~b}$ is small $(\approx 2[i n])$. Therefore, we get for the ratio of the maximum deflections the estimate:

$$
20 \leq \frac{\delta_{1}}{\delta_{2}} \leq 26
$$

Consequently, we conclude that the deflection $\delta_{1}$ is significantly larger than the deflection $\delta_{2}$. This conclusion also applies to the moment arm corrections. We get:

$$
\left|\delta_{1}\right| \gg\left|\delta_{2}\right| \quad \Longrightarrow \quad\left|l_{1}\right| \gg\left|l_{2}\right| \quad \Longrightarrow \quad l_{2} \approx 0
$$


Finally, after (i) replacing $l_{1}$ and $l_{2}$ in Eq. (22) using Eq. (24) and the result of Eq. (26), and after (ii) using the symbol $\delta$ instead of $\delta_{1}$, we get for the total moment arm correction the equation:

$$
\Delta l=\frac{h}{l_{\circ} / \cos \phi} \cdot \delta
$$

An estimate of the beam deflection $\delta$ due to a concentrated force is still needed so that the coefficients of the theoretical regression model of the sum of the gage outputs can be quantified. The deflection can be found using the Area-Moment Method (see Ref. [8] for more detail). Then, the deflection equals

$$
\delta=\int_{a}^{b} \frac{M(x) \cdot x}{E(x) \cdot I(x)} d x
$$

where $x$ is the beam coordinate, $a$ and $b$ are the coordinate of the fixed and free end of the beam, $M(x)$ is the local moment, $E(x)$ is the modulus of elasticity, and $I(x)$ is the moment of inertia of the cross-section of the beam. The modulus of elasticity and the moment of inertia need to be moved in front of the integral symbol so that the deflection can be estimated. This can be done by using a generalized form of the Mean Value Theorem of the Integral Calculus (see Ref. [9], pp.126-128). The theorem can be described as follows:

If $f(x)$ and $p(x)$ are continuous functions in $a \leq x \leq b$, and $p(x) \geq 0$ or, more general, $p(x)$ does not change sign in the interval $a \leq x \leq b$, then

$$
\int_{a}^{b} f(x) \cdot p(x) d x=f(\xi) \cdot \int_{a}^{b} p(x) d x
$$

where $\xi$ is some constant that fulfills the condition $a \leq \xi \leq b$.

The following four substitutions have to be made so that Eq. (29) may be applied to Eq. (28):

$$
\begin{aligned}
a & =0 \\
b & =l_{\circ} / \cos \phi \\
f(x) & =\frac{1}{E(x) \cdot I(x)} \\
p(x) & =M(x) \cdot x
\end{aligned}
$$

Then, Eq. (28) can be written in the following form:

$$
\delta=\frac{1}{E(\xi) \cdot I(\xi)} \cdot \int_{0}^{l_{0} / \cos \phi} M(x) \cdot x d x
$$

From Fig. 9a we conclude that the following equation is valid:

$$
M(x) \cdot x=[F \cdot \cos \phi \cdot x] \cdot x
$$

In the next step, after replacing the integrand in Eq. (31) by the right hand side of Eq. (32) and knowing that $F \cdot \cos \phi$ is a constant concentrated load at the assumed free end of a cantilever beam, we get:

$$
\delta=\frac{F \cdot \cos \phi}{E(\xi) \cdot I(\xi)} \cdot \int_{0}^{l_{\circ} / \cos \phi} x^{2} d x
$$


Finally, after solving the integral in Eq. (33a) analytically, we get the following value for the deflection of the sting balance:

$$
\delta=\frac{F \cdot l_{\circ}^{3}}{3 \cdot E(\xi) \cdot I(\xi) \cdot \cos ^{2} \phi}
$$

Now, it is possible to get the moment arm correction due to bending. It is only required (i) to replace the deflection $\delta$ in Eq. (27) with the right hand side of Eq. (33b). Then, the moment arm correction becomes:

$$
\Delta l=\frac{h}{l_{\circ} / \cos \phi} \cdot \delta=\frac{h}{l_{\circ} / \cos \phi} \cdot \frac{F \cdot l_{\circ}^{3}}{3 \cdot E(\xi) \cdot I(\xi) \cdot \cos ^{2} \phi}
$$

Equation (34) can be simplified further. After some algebra we get for the moment arm correction:

$$
\Delta l=\frac{1}{E(\xi) \cdot I(\xi)} \cdot\left(\frac{h}{3}\right) \cdot\left(\frac{1}{\cos \phi}\right) \cdot F \cdot l_{\circ}^{2}
$$

\section{Approximated Solution of Moment Arm Correction}

Unfortunately, the numerical value of the moment arm correction due to bending cannot easily be computed using Eq. (35) as the exact value of $E(\xi) \cdot I(\xi)$ is unknown. However, it is possible to develop an estimate for $E(\xi) \cdot I(\xi)$ by using an alternate expression for the deflection of a cantilever beam. From Ref. [8], p.159, we also know that

$$
\frac{d \Theta}{d x}=\frac{M(x)}{E(x) \cdot I(x)}
$$

where $\Theta$ is the deflection angle. Then, Eq. (28) can also be written as follows:

$$
\delta=\int_{a}^{b} \frac{M(x) \cdot x}{E(x) \cdot I(x)} d x=\int_{a}^{b} \frac{d \Theta}{d x} \cdot x d x
$$

In most cases the slope $d \Theta / d x$ is a function of the beam coordinate $x$. A simplifying assumption, however, can be made that will make it possible to assess the magnitude of the coefficients of the regression model of the sum of the gage output. It is simply assumed that the slope $d \Theta / d x$ is constant:

$$
\frac{d \Theta}{d x} \approx \text { constant } \approx \frac{\Theta(b)-\Theta(a)}{b-a}
$$

Then, the deflection of the cantilever beam at the free end can be approximated as follows:

$$
\delta \approx \frac{\Theta(b)-\Theta(a)}{b-a} \cdot \int_{a}^{b} x d x=\frac{\Theta(b)-\Theta(a)}{b-a} \cdot \frac{b^{2}-a^{2}}{2}=[\Theta(b)-\Theta(a)] \cdot \frac{a+b}{2}
$$

Equation (39) may be applied to the sting balance if the following assumptions are made:

$$
a=0 ; b=l_{\circ} / \cos \phi ; \Theta(a)=0 ; \Theta(b) \Longrightarrow \text { from experiment }
$$

Then, the deflection at the free end becomes:

$$
\delta \approx l_{\circ} / \cos \phi \cdot \frac{\Theta\left(l_{\circ} / \cos \phi\right)}{2}
$$

Now, after replacing $\delta$ in Eq. (33b) with the right hand side of Eq. (40), Eq. (33b) becomes:

$$
l_{\circ} / \cos \phi \cdot \frac{\Theta\left(l_{\circ} / \cos \phi\right)}{2} \approx \frac{F \cdot l_{\circ}^{3}}{3 \cdot E(\xi) \cdot I(\xi) \cdot \cos ^{2} \phi}
$$


After some algebra we get for the inverse of the product of the modulus of elasticity with the moment of inertia the following approximation:

$$
\frac{1}{E(\xi) \cdot I(\xi)} \approx \frac{3 \cdot \Theta\left(l_{\circ} / \cos \phi\right)}{2 \cdot F \cdot l_{\circ} \cdot l_{\circ} / \cos \phi}
$$

From Fig. 8 we also know that:

$$
l_{\circ} / \cos \phi=\sqrt{h^{2}+l_{\circ}^{2}}
$$

Then, using Eq. (20) to replace $F \cdot l_{\circ}$ in Eq. (42) and after replacing $l_{\circ} / \cos \phi$ with the right hand side of Eq. (43), Eq. (42) can be written as:

$$
\frac{1}{E(\xi) \cdot I(\xi)} \approx \frac{3 \cdot \Theta\left(l_{\circ} / \cos \phi\right)}{2 \cdot M_{\circ} \sqrt{h^{2}+l_{\circ}^{2}}}
$$

Fortunately, the deflection angle at the free end as a function of the applied loads was determined during the preparation of the sting balance for a test at Ames Research Center. This data was fitted using a least squares fit and the following regression model of the deflection angle at the free end was obtained:

$$
\Theta\left(l_{\mathrm{o}} / \cos \phi\right) \approx\left[k_{1} \cdot M_{\mathrm{o}}+k_{2} \cdot F\right] \cdot \frac{\pi}{180^{\circ}} \quad \Longrightarrow \quad \text { from experiment }
$$

where

$$
\begin{aligned}
& k_{1}=+0.00329615\left[\frac{d e g}{i n-l b s}\right] \\
& k_{2}=-0.01702055\left[\frac{d e g}{l b s}\right]
\end{aligned}
$$

The force $F$ in Eq. (45a) can be replaced by using the "nominal" moment and moment arm. We get:

$$
F=\frac{M_{\circ}}{l_{\circ}}
$$

Then, Eq. (45a) can be written in the following form:

$$
\Theta\left(l_{\circ} / \cos \phi\right) \approx\left[k_{1} \cdot M_{\circ}+\left(k_{2} / l_{\circ}\right) \cdot M_{\circ}\right] \cdot \frac{\pi}{180^{\circ}}=\left[k_{1}+\left(k_{2} / l_{\circ}\right)\right] \cdot \frac{\pi}{180^{\circ}} \cdot M_{\circ}
$$

Equation (47) may be simplified further. The range of the "nominal" moment arm is known from balance calibration records. Therefore, the following relationship applies:

$$
16.52[i n] \leq l_{\circ} \leq 25.92[i n]
$$

Now, using the result of Eq. (48) with Eq. (45c), we get the following estimate:

$$
0.00066\left[\frac{d e g}{i n-l b s}\right] \leq\left|k_{2} / l_{\circ}\right| \leq 0.00103\left[\frac{d e g}{i n-l b s}\right]
$$

Comparing the absolute value of the coefficient $k_{1}$ given in Eq. (45b) with the range of the absolute value of the term $k_{2} / l_{\circ}$ we see that $\left|k_{1}\right|$ is three to five times larger than the term $\left|k_{2} / l_{\circ}\right|$. Therefore, we can make the following simplifying assumption:

$$
\left|k_{1}\right| \gg\left|k_{2} / l_{\circ}\right| \Longrightarrow\left|k_{2} / l_{\circ}\right| \approx 0
$$

Then, using the result of Eq. (50), Eq. (47) becomes:

$$
\Theta\left(l_{\mathrm{o}} / \cos \phi\right) \approx k_{1} \cdot \frac{\pi}{180^{\circ}} \cdot M_{\mathrm{o}}
$$


Finally, after using the result of Eq. (51) in Eq. (44), we get:

$$
\frac{1}{E(\xi) \cdot I(\xi)} \approx \frac{k_{1} \cdot \pi}{120^{\circ} \cdot \sqrt{h^{2}+l_{\circ}^{2}}}
$$

Now, using the result of Eq. (52) in Eq. (35), we get for the moment arm correction the equation:

$$
\Delta l \approx \frac{k_{1} \cdot \pi \cdot h}{360^{\circ} \cdot \sqrt{h^{2}+l_{\mathrm{o}}^{2}}} \cdot\left(\frac{1}{\cos \phi}\right) \cdot F \cdot l_{\mathrm{o}}^{2}
$$

We also know from Fig. 8 that the following relationship applies:

$$
\sin \phi=\frac{h}{\sqrt{h^{2}+l_{\circ}^{2}}}
$$

Therefore, Eq. (53) can be expressed as follows:

$$
\Delta l \approx \frac{k_{1} \cdot \pi}{360^{\circ}} \cdot \tan \phi \cdot F \cdot l_{\circ}^{2}
$$

\section{Approximated Solution of Exact Regression Model}

Finally, the moment arm correction estimate defined in Eq. (55) is inserted into Eq. (21). Then, the sum of the gage outputs becomes:

$$
R_{1}+R_{2} \approx[\gamma \cdot 2] \cdot M_{\circ}+\left[\frac{\gamma \cdot k_{1} \cdot \pi}{180^{\circ}} \cdot \tan \phi\right] \cdot F^{2} \cdot l_{\circ}^{2}
$$

The numerical value of $\tan \phi$ in Eq. (56) depends on the "nominal" moment arm $l_{\circ}$. We know that

$$
\tan \phi \quad \Longrightarrow \tan \phi\left(l_{\circ}\right)
$$

We can use again the definition of the "nominal" moment (Eq. (20)). Then, after substituting $F \cdot l_{\circ}$ in Eq. (56) using $M_{\mathrm{o}}$, we get the final form of a theoretical regression model of the sum of the gage outputs:

$$
\begin{aligned}
R_{1}+R_{2} & \approx \eta_{1}^{\prime} \cdot M_{\circ}+\eta_{2}^{\prime} \cdot M_{\circ}^{2} \\
\eta_{1}^{\prime} & =\gamma \cdot 2 \\
\eta_{2}^{\prime} & =\frac{\gamma \cdot k_{1} \cdot \pi}{180^{\circ}} \cdot \tan \phi\left(l_{\circ}\right)
\end{aligned}
$$

In the next part of the paper the theoretical regression coefficients of the regression models for the difference and the sum of the gage outputs are compared with results of the optimized regression models.

\section{Regression Coefficient Comparison}

\section{A. General Remarks}

In the previous sections exact and approximate solutions of the regression models of (i) the difference and (ii) the sum of the gage outputs of the sting balance were developed. The theoretical and fitted values of the regression model coefficients need to be compared in order (i) to assess the validity of the assumptions 
that were used to develop the theoretical regression models and (ii) to arrive at a better understanding of the calibration data. The theoretical values of the regression coefficients are computed using balance geometry data and other information. The regression cofficients of the optimized regression models, on the other hand, are taken directly from the listed regression analysis results (column three in Figs. 7a and 7b).

\section{B. Difference of Gage Outputs}

At first, the optimized regression model of the difference of the gage outputs is investigated. The optimized regression model is defined in Eq. (2c). The parameter $d$, i.e., the distance between the two moment gages of the sting balance is needed in order to determine the theoretical value of the first coefficient $\epsilon_{1}$ that is related to the applied calibration force $F$. The theoretical value of the coefficient is defined in Eq. (14b). From engineering drawings of the sting balance we know that the parameter $d$ is given as:

$$
d=5.5[i n]
$$

Then, using the estimate for $\gamma$ given in Eq. (11b) and the value of the distance $d$ given in Eq. (59), the theoretical value of the coefficient becomes:

Theoretical Analysis: $\epsilon_{1}^{\prime}=\gamma \cdot d=11.9400[(\mu V / V) / l b s]$

The corresponding coefficient value for the optimized regression model is listed in the table in Fig. 7a (column three). The coefficient has the following value:

$$
\text { Least Squares Fit: } \quad \epsilon_{1}=12.2069[(\mu V / V) / l b s]
$$

Comparing the right hand side of Eq. (60a) with the right hand side of Eq. (60b) we see that the theoretical and the fitted value show excellent agreement.

\section{Sum of Gage Outputs}

In the next step the coefficients of the optimized regression model of the sum of the gage outputs needs to be compared. The optimized regression model is defined in Eq. (3c). At first, the coefficient of the linear term is investigated. Its theoretical value is given in Eq. (58b). It has the following solution:

$$
\text { Theoretical Analysis: } \quad \eta_{1}^{\prime}=\gamma \cdot 2=4.3418[(\mu V / V) /(i n-l b s)]
$$

The corresponding coefficient value for the optimized regression model can be found in the third column in Fig. 7b. It is given as:

$$
\text { Least Squares Fit : } \quad \eta_{1}=4.3446[(\mu V / V) /(i n-l b s)]
$$

Again, after comparing the right hand side of Eq. (61a) with the right hand side of Eq. $(61 b)$, we see that the theoretical and the fitted values show excellent agreement.

It remains to compare the coefficient of the square term of the regression model of the sum of the gage output. The estimated theoretical value of the term is given on the right hand side of Eq. (58c). Unfortunately, the angle $\phi$ is not constant for the calibration data set. Therefore, the range of the magnitude of the theoretical value of the coefficient needs to be computed as a function of the range of angle $\phi$. From 
the calibration records we know that the range of angle $\phi$ is given as follows:

$$
15^{\circ} \leq \phi\left(l_{\circ}\right) \leq 20^{\circ}
$$

Therefore, $\tan \phi$ has the following range:

$$
0.27 \leq \tan \phi\left(l_{\circ}\right) \leq 0.36
$$

Consequently, after using (i) the range given in Eq. (62b) and (ii) the values for $\gamma$ and $k_{1}$ given in Eq. (11b) and Eq. (45b) in Eq. (58c), we get the following range for the theoretical value of the second coefficient of the regression model of the sum of the gage outputs:

Theoretical Analysis : $\quad 3.4 \times 10^{-5}\left[\frac{(\mu V / V)}{(i n-l b s)^{2}}\right] \leq \eta_{2}^{\prime} \leq 4.5 \times 10^{-5}\left[\frac{(\mu V / V)}{(i n-l b s)^{2}}\right]$

Again, the corresponding value of the second coefficient of the optimized regression model can be found in the third column in Fig. 7b. It is given as:

$$
\text { Least Squares Fit : } \quad \eta_{2}=-7.3 \times 10^{-6}\left[\frac{(\mu V / V)}{(i n-l b s)^{2}}\right]
$$

Two observations can be made if the range of the theoretical value (Eq. (63a)) is compared with the least squares estimate (Eq. (63b)): (1) the absolute value of the theoretical value is approximately four to six times larger than the fitted value; (2) the sign of the theoretical value does not equal the sign of the fitted value. How can these differences between the theoretical estimate and the fitted value of the second coefficient be explained? It must be remembered that the calculation of the theoretical estimate did not take the fact into account that the calibration fixture was leveled each time after a specific calibration load was applied. Figure 10 shows the impact of the leveling of the calibration fixture on the moment arm. Several observations can be made using Fig. 10:

Observation 1: The leveling of the balance partially counteracts the increase of the moment arm due to the elastic deformation of the sting balance under load. Therefore, the magnitude of the fitted coefficient must be significantly smaller than the magnitude of a theoretical estimate of the coefficient as the theoretical estimate does not account for the leveling of the calibration fixture.

Observation 2: The final position of the force attachment point (i.e., position 3 in Fig. 10) does no longer coincide with the original position of the load attachment point (i.e., position 1 in Fig. 10) because the sting balance remains elastically deformed even though the calibration fixture was leveled.

Observation 3: The unloaded sting is defined using the line $A B C$ in Fig. 10. This line was also used to define the "nominal" moment arm $l_{\circ}$ that is used to compute the "nominal" moment $M_{\circ}$. The loaded sting (after leveling) is defined using the line $A^{\prime} B^{\prime} C$. The leveling removed most of the moment arm change due to sting bending. In addition, due to the remaining elastic deformation of the sting under load, the sting segments $A^{\prime} B^{\prime}$ and $B^{\prime} C$ are no longer straight lines. Therefore, the total length of the moment arm must have actually been reduced after the calibration fixture was leveled under load. This explains the observation that the sign of the fitted second coefficient of the regression model is negative and not positive. The sign of the coefficient would have been positive if the leveling of the calibration fixture would not have removed the moment arm change due to sting bending.

\section{Summary and Conclusions}

A regression model optimization algorithm was applied to wind tunnel sting balance calibration data. The optimization algorithm correctly predicted that (i) the difference of the gage outputs should be modeled 
using an intercept term and the normal force at the BMC and that (ii) the sum of the gage outputs should be modeled using an intercept term, the moment at the BMC, and the square of the moment at the BMC. It was also shown that the difference and the sum of the gage outputs best describe the physical characteristics of a sting balance if the normal force and the moment at the BMC are the chosen calibration loads.

Classical strain-gage balance load transformations were applied in order to demonstrate that the optimized regression model of the difference of the gage outputs is supported by a rigorous analysis of the physics of the balance. The theoretical analysis of the relationship between the loads at the BMC and the sum of the gage outputs was more difficult. In that case, the equations of the deflection of a cantilever beam had to be applied in order to confirm the validity of the optimized regression model. It was also demonstrated that the leveling of the sting balance during the calibration is important. It removes most of the moment arm change that is introduced in the calibration data set due to the bending of the sting balance under load. The analysis also showed that the remaining elastic deformation of the leveled sting balance is mostly responsible for the presence of the square of the moment in the regression model of the sum of the gage outputs.

In conclusion, the regression analysis of the sting balance calibration data set is a rare example of a situation when the regression model of a strain-gage balance calibration data set can be derived from first principles of physics and engineering. It is also interesting to note that the regression model optimization algorithm predicted the same math term combination for the balance calibration data using only a set of statistical quality metrics.

\section{Acknowledgements}

The authors would like to thank Thomas R. Wayman of The Gulfstream Aerospace Corporation for providing the sting balance calibration data set that was used in the study. Thanks also go to Max Amaya of NASA Ames and Tom Volden of Jacobs Technology for their critical and constructive review of the final manuscript. The work reported in this paper was partially supported by NASA's Aeronautic Test Program and the Wind Tunnel Division at Ames Research Center under contract NNA04BA85C.

\section{References}

${ }^{1}$ Ulbrich, N., "Regression Model Optimization for the Analysis of Experimental Data," AIAA 2009-1344, paper presented at the 47th AIAA Aerospace Sciences Meeting and Exhibit, Orlando, Florida, January 2009.

${ }^{2}$ Ulbrich, N. and Volden, T., "Regression Analysis of Experimental Data Using an Improved Math Model Search Algorithm," AIAA 2008-0833, paper presented at the 46th AIAA Aerospace Sciences Meeting and Exhibit, Reno, Nevada, January 2008.

${ }^{3}$ Ulbrich, N. and Volden, T., "Strain-Gage Balance Calibration Analysis Using Automatically Selected Math Models," AIAA 2005-4084, paper presented at the 41st AIAA/ASME/SAE/ASEE Joint Propulsion Conference and Exhibit, Tucson, Arizona, July 2005.

${ }^{4}$ Ulbrich, N. and Volden, T., "Application of a New Calibration Analysis Process to the MK-III-C Balance," AIAA 2006-0517, paper presented at the 44th AIAA Aerospace Sciences Meeting and Exhibit, Reno, Nevada, January 2006.

${ }^{5}$ Ulbrich, N. and Volden, T., "Analysis of Floor Balance Calibration Data using Automatically Generated Math Models," AIAA 2006-3437, paper presented at the 25th AIAA Aerodynamic Measurement Technology and Ground Testing Conference, San Francisco, California, June 2006.

${ }^{6}$ Ulbrich, N. and Volden, T., "Analysis of Balance Calibration Machine Data using Automatically Generated Math Models," AIAA 2007-0145, paper presented at the 45th AIAA Aerospace Sciences Meeting and Exhibit, Reno, Nevada, January 2007.

${ }^{7}$ Ulbrich, N., Volden, T., and Booth, D., "Predictive Capabilities of Regression Models used for StrainGage Balance Calibration Analysis," AIAA 2008-4028, paper presented at the 26th AIAA Aerodynamic Measurement Technology and Ground Testing Conference, Seattle, Washington, June 2008.

${ }^{8}$ Timoshenko, S. and MacCullough, G. H., Elements of Strength of Materials, D. Van Nostrand Company, Inc., Toronto, New York, London, 2nd ed., 1940, pp.160-170.

${ }^{9}$ Courant, R., Differential and Integral Calculus, Vol. 1, 2nd ed., Interscience Publishers, John Wiley \& Sons, Inc., New York, 1937, reprinted 1962, pp.126-128. 


\section{Appendix: Classical Strain-Gage Balance Load Transformations}

\section{A. Introduction}

Different types of strain-gage balance designs are used in wind tunnel testing. A force type balance, for example, is designed such that a balance gage responds to a force at the gage location. A moment type balance, on the other hand, has gages that respond to a moment at the gage location. An alternate derivation of the classical load transformation equations for these two types of balances is presented in this appendix. These transformation equations describe the relationship between loads at the balance moment center (BMC) and loads at the location of the gages.

\section{B. Force Type Balance}

Figure 11 shows forces and moments that act on a typical strain-gage balance. The load transformation equations for a force type balance can easily be derived if the force $F$ and moment $M$ at the BMC are replaced by two forces $F_{1}$ and $F_{2}$ at the location of the gages. The sum of these two forces equals the force at the BMC. We get:

$$
F=F_{1}+F_{2}
$$

Similarly, we know that the total moment at the BMC equals the sum of the moment contributions from the two forces that replace the force and moment at the BMC. It is assumed that the location of the $\mathrm{BMC}$ and the location of the gages are described in a coordinate system that uses the balance centerline as the coordinate system axis (see Fig. 11). Then, the BMC has the coordinate $\mu$, the center of the forward gage the coordinate $c_{1}$, and the center of the aft gage the coordinate $c_{2}$. Now, two coordinate differences $d_{1}$ and $d_{2}$ may be defined that describe the distance between the BMC and the forces $F_{1}$ and $F_{2}$ at the balance gage locations. We get:

$$
\begin{aligned}
& d_{1}=\mu-c_{1} \\
& d_{2}=\mu-c_{2}
\end{aligned}
$$

Then, using the sign definitions of (i) the balance loads and of (ii) the coordinates that are depicted in Fig. 11, the total moment at the BMC can be expressed as follows:

$$
M=F_{1} \cdot d_{1}-F_{2} \cdot\left(-d_{2}\right)
$$

From Eq. (A.1) we know that:

$$
F_{2}=F-F_{1}
$$

The right hand side of Eq. (A.4) may be used to replace the force $F_{2}$ in Eq. (A.3). We get:

$$
M=F_{1} \cdot d_{1}-\left(F-F_{1}\right) \cdot\left(-d_{2}\right)
$$

Rearranging terms in Eq. (A.5) and after some algebra we get:

$$
F_{1}=F \cdot \frac{\left(-d_{2}\right)}{d_{1}-d_{2}}+M \cdot \frac{1}{d_{1}-d_{2}}
$$

Similarly, using Eq. (A.6) to replace force $F_{1}$ in Eq. (A.4) and after some algebra, we get:

$$
F_{2}=F \cdot \frac{d_{1}}{d_{1}-d_{2}}-M \cdot \frac{1}{d_{1}-d_{2}}
$$

Finally, using Eqs. $(A .2 a)$ and $(A .2 b)$ to replace the moment arms in Eqs. $(A .3),(A .6)$, and $(A .7)$, we get the set of load transformation equations for a force type balance: 


\section{LOAD TRANSFORMATIONS - FORCE TYPE BALANCE}

(univeral relationships - valid for any given balance moment center location)

$$
\begin{aligned}
F & =F_{1}+F_{2} \\
M & =F_{1} \cdot\left(\mu-c_{1}\right)-F_{2} \cdot\left(c_{2}-\mu\right) \\
F_{1} & =F \cdot \frac{c_{2}-\mu}{c_{2}-c_{1}}+M \cdot \frac{1}{c_{2}-c_{1}} \\
F_{2} & =F \cdot \frac{\mu-c_{1}}{c_{2}-c_{1}}-M \cdot \frac{1}{c_{2}-c_{1}}
\end{aligned}
$$

\section{Moment Type Balance}

A moment type balance is designed such that a balance gage responds to a moment at the gage location. Therefore, we need to express the moment at the gage location as a function of the force and moment at the $\mathrm{BMC}$ in order to derive the desired load transformation equations. We know from Fig. 12 that the moment $M_{1}$ at the forward gage is caused by the force $F_{2}$ at the aft gage. Therefore, using the gage coordinates defined in Fig. 12, we can write:

$$
M_{1}=(-1) \cdot F_{2} \cdot\left(c_{2}-c_{1}\right)
$$

Now, using the right hand side of Eq. $(A .8 d)$ to replace the force $F_{2}$ in Eq. $(A .9)$, we get:

$$
M_{1}=(-1) \cdot\left(F \cdot \frac{\mu-c_{1}}{c_{2}-c_{1}}-M \cdot \frac{1}{c_{2}-c_{1}}\right) \cdot\left(c_{2}-c_{1}\right)
$$

Finally, after simplifying and rearranging the right hand side of Eq. (A.10), we get:

$$
M_{1}=M-F \cdot\left(\mu-c_{1}\right)
$$

The derivation of the moment $M_{2}$ at the aft gage uses the same approach. This time, the moment $M_{2}$ at the aft gage is caused by the force $F_{1}$ at the forward gage. We get:

$$
M_{2}=(+1) \cdot F_{1} \cdot\left(c_{2}-c_{1}\right)
$$

Now, using the right hand side of Eq. $(A .8 c)$ to replace the force $F_{1}$ in Eq. $(A .12)$, we get:

$$
M_{2}=(+1) \cdot\left(F \cdot \frac{c_{2}-\mu}{c_{2}-c_{1}}+M \cdot \frac{1}{c_{2}-c_{1}}\right) \cdot\left(c_{2}-c_{1}\right)
$$

Finally, after simplifying and rearranging the right hand side of Eq. (A.13), we get:

$$
M_{2}=M+F \cdot\left(c_{2}-\mu\right)
$$

The remaining transformations can be obtained from Eqs. (A.11) and (A.14). Subtracting the left and right hand side of Eq. (A.11) from the left and right hand side of Eq. $(A .14)$, we get:

$$
M_{2}-M_{1}=F \cdot\left(c_{2}-c_{1}\right)
$$

Then, after solving Eq. (A.15) for the force at the BMC, we get the following relationship:

$$
F=\frac{M_{2}-M_{1}}{c_{2}-c_{1}}
$$


In addition, after solving Eq. (A.11) for the moment at the BMC, we also know that:

$$
M=M_{1}+F \cdot\left(\mu-c_{1}\right)
$$

Then, using the right hand side of Eq. (A.16) to replace the force $F$ in Eq. (A.17), we get:

$$
M=M_{1}+\left(\frac{M_{2}-M_{1}}{c_{2}-c_{1}}\right) \cdot\left(\mu-c_{1}\right)
$$

We also know that the right hand side of Eq. (A.18) can be written in the following form:

$$
M_{1}+\left(\frac{M_{2}-M_{1}}{c_{2}-c_{1}}\right) \cdot\left(\mu-c_{1}\right)=M_{1} \cdot\left(\frac{c_{2}-c_{1}}{c_{2}-c_{1}}\right)+M_{2} \cdot\left(\frac{\mu-c_{1}}{c_{2}-c_{1}}\right)-M_{1} \cdot\left(\frac{\mu-c_{1}}{c_{2}-c_{1}}\right)
$$

Now, after (i) simplifying the right hand side of Eq. (A.19) and after (ii) replacing the right hand side of Eq. (A.18) with the result, we get for the moment $M$ at the BMC:

$$
M=M_{1} \cdot\left(\frac{c_{2}-\mu}{c_{2}-c_{1}}\right)+M_{2} \cdot\left(\frac{\mu-c_{1}}{c_{2}-c_{1}}\right)
$$

Finally, the set of load transformation equations for a moment type balance can be summarized:

\section{LOAD TRANSFORMATIONS - MOMENT TYPE BALANCE}

(univeral relationships - valid for any given balance moment center location)

$$
\begin{aligned}
F & =\frac{M_{2}-M_{1}}{c_{2}-c_{1}} \\
M & =M_{1} \cdot\left(\frac{c_{2}-\mu}{c_{2}-c_{1}}\right)+M_{2} \cdot\left(\frac{\mu-c_{1}}{c_{2}-c_{1}}\right) \\
M_{1} & =M-F \cdot\left(\mu-c_{1}\right) \\
M_{2} & =M+F \cdot\left(c_{2}-\mu\right)
\end{aligned}
$$

\section{Load Transformation Equations for Simplified Gage Configuration}

The BMC is often located halfway between the forward and aft gage of a balance. In that case, the load transformation equations can be simplified significantly if the gage distance $d$ is introduced as a parameter. Then, after placing the coordinate system origin at the location of the forward gage, we get:

$$
\begin{aligned}
c_{1} & =0 \\
c_{2} & =d \\
\mu & =d / 2
\end{aligned}
$$

Now, after applying Eqs. $(A .22 a),(A .22 b),(A .22 c)$ to Eqs. $(A .8 a),(A .8 b),(A .8 c),(A .8 d)$ and after some algebra, we get the simplified transformations for the force type balance: 


\section{SIMPLIFIED LOAD TRANSFORMATIONS - FORCE TYPE BALANCE}

(valid if the balance moment center is located halfway between the forward and aft gage)

$$
\begin{aligned}
F & =F_{1}+F_{2} \\
M & =\frac{F_{1}-F_{2}}{2} \cdot d \\
F_{1} & =\frac{F}{2}+\frac{M}{d} \\
F_{2} & =\frac{F}{2}-\frac{M}{d}
\end{aligned}
$$

Similarly, after applying Eqs. $(A .22 a),(A .22 b),(A .22 c)$ to Eqs. $(A .21 a),(A .21 b),(A .21 c),(A .21 d)$ and after some algebra, we get the simplified transformations for the moment type balance:

\section{SIMPLIFIED LOAD TRANSFORMATIONS - MOMENT TYPE BALANCE}

(valid if the balance moment center is located halfway between the forward and aft gage)

$$
\begin{aligned}
F & =\frac{M_{2}-M_{1}}{d} \\
M & =\frac{M_{1}+M_{2}}{2} \\
M_{1} & =M-\frac{F}{2} \cdot d \\
M_{2} & =M+\frac{F}{2} \cdot d
\end{aligned}
$$




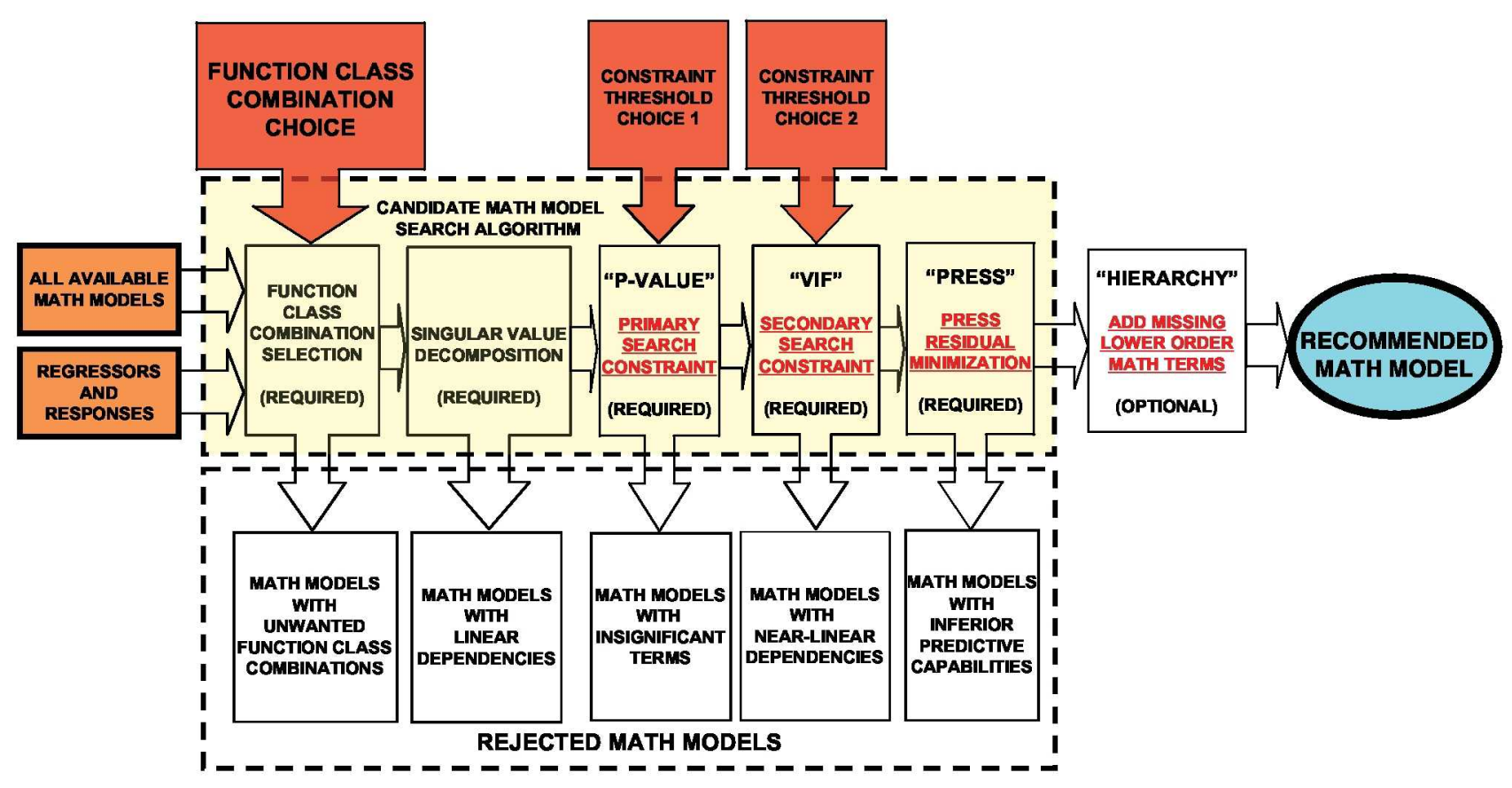

Fig. 1 Key elements of candidate math model search algorithm.

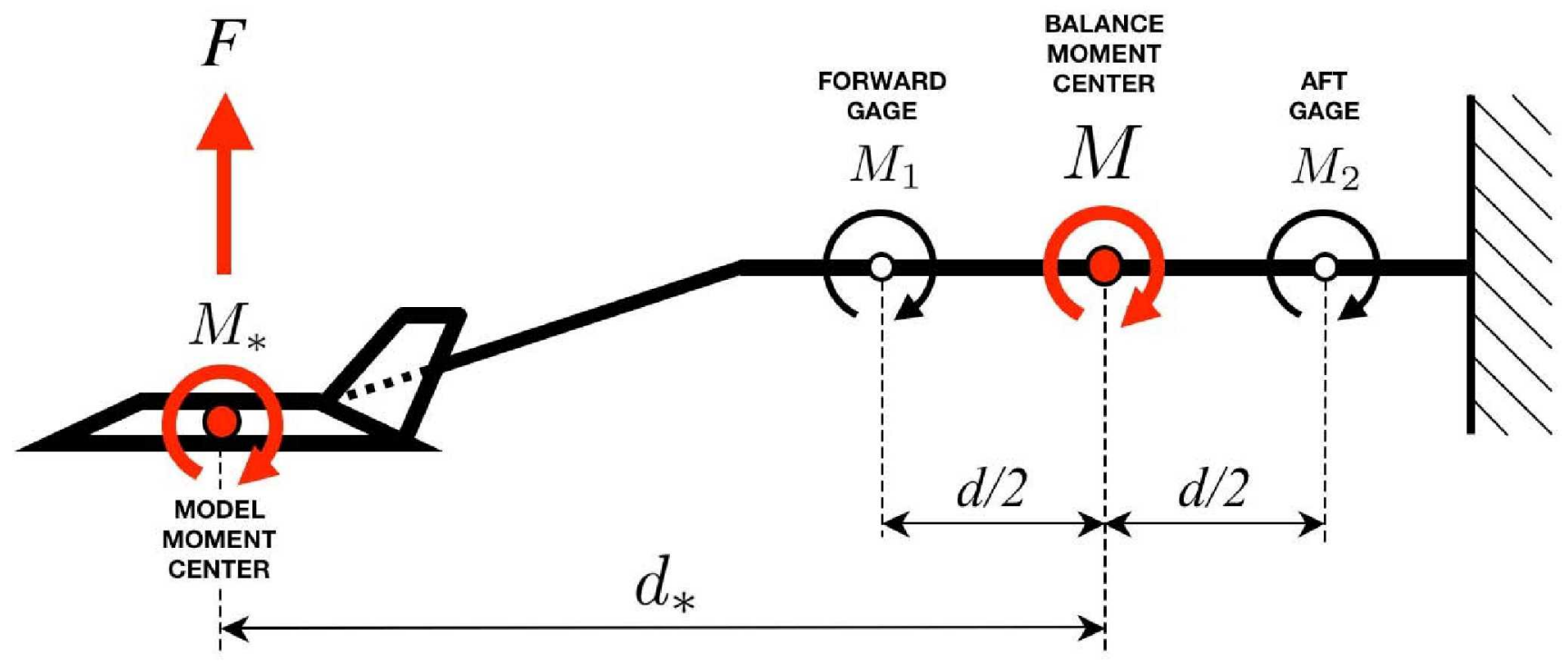

Fig. 2 Forces and moments acting at different locations on a wind tunnel sting balance. 


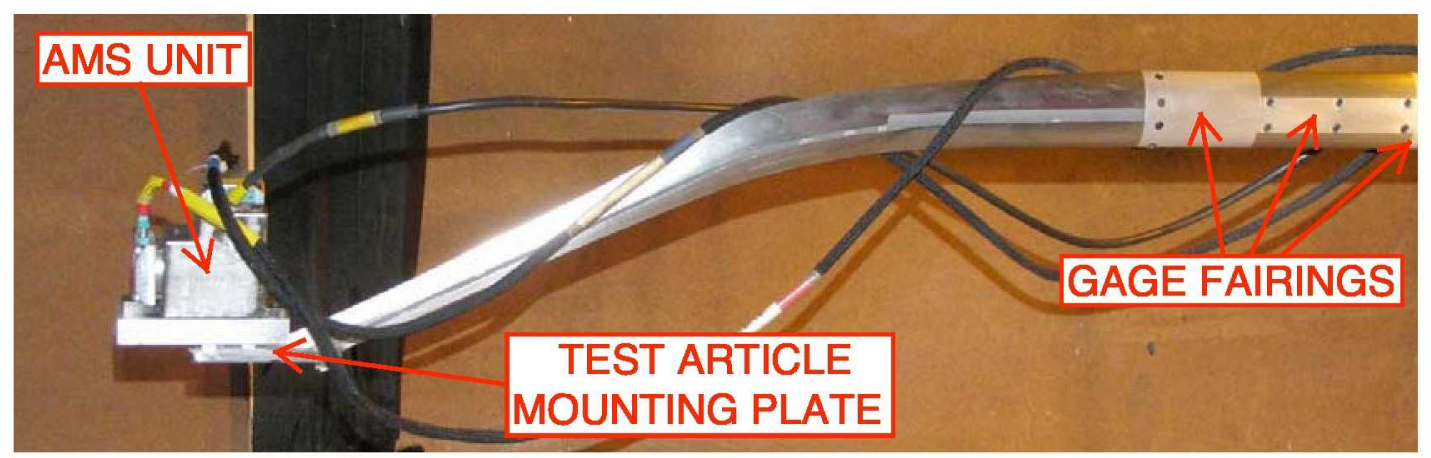

Fig. 3a Sideview of sting balance with attached AMS (angle measurement system) unit. (Courtesy of The Gulfstream Aerospace Corporation, Savannah, Georgia.)

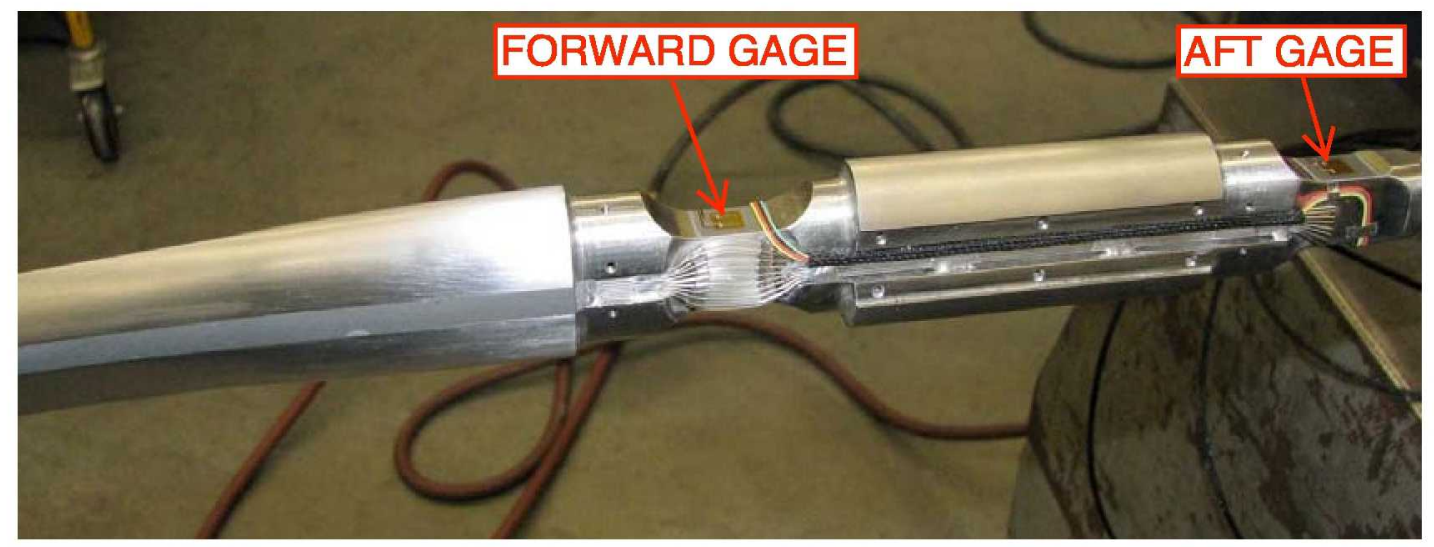

Fig. 3b Location of forward and aft gage of sting balance after removal of gage fairings. (Courtesy of The Gulfstream Aerospace Corporation, Savannah, Georgia.)

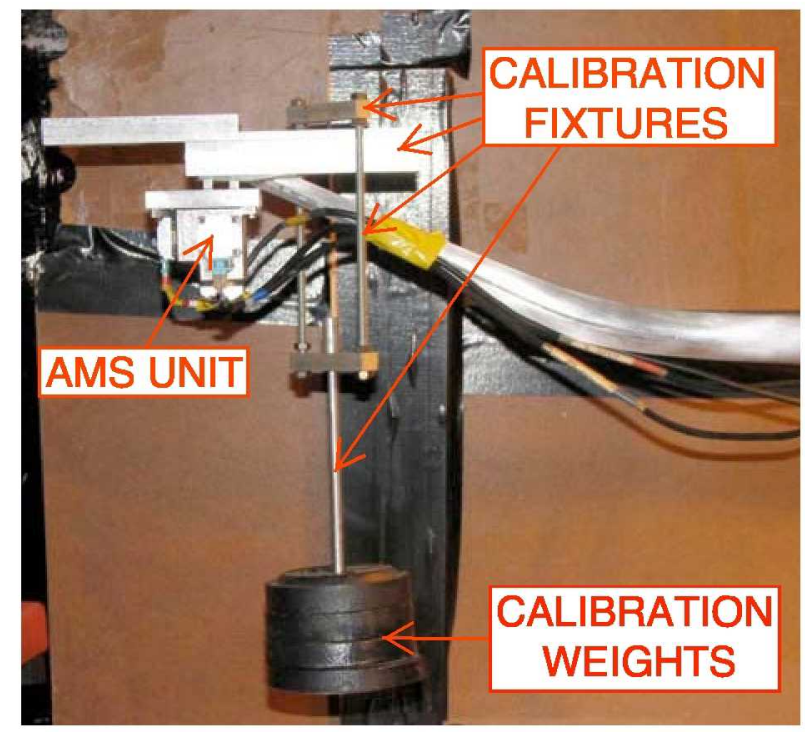

Fig. 3c Calibration of sting balance in upside-down orientation using weights.

(Courtesy of The Gulfstream Aerospace Corporation, Savannah, Georgia.) 


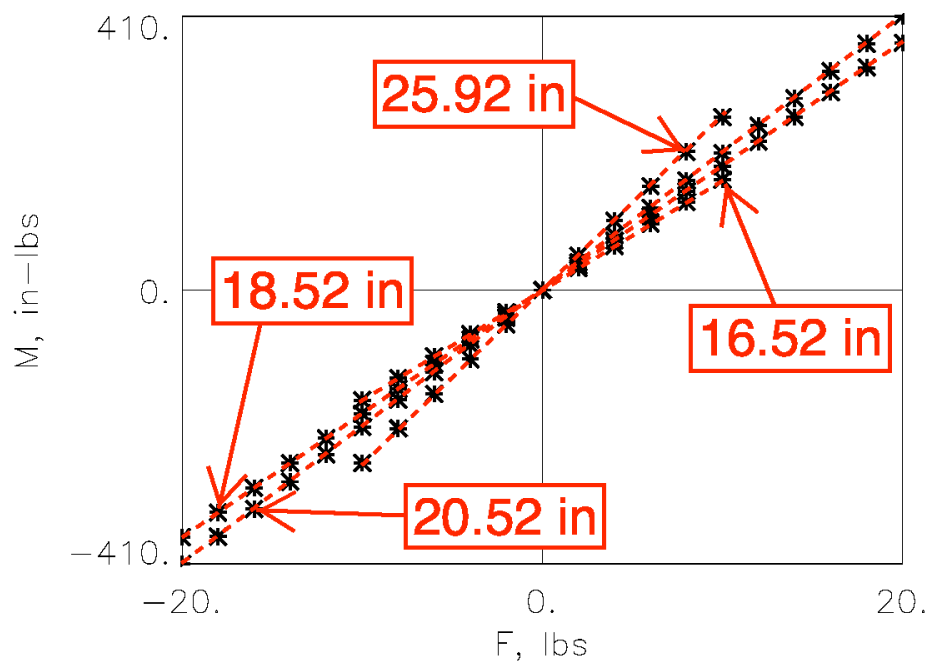

Fig. 4 Calibration forces and moments at the balance moment center for the four selected moment arms.

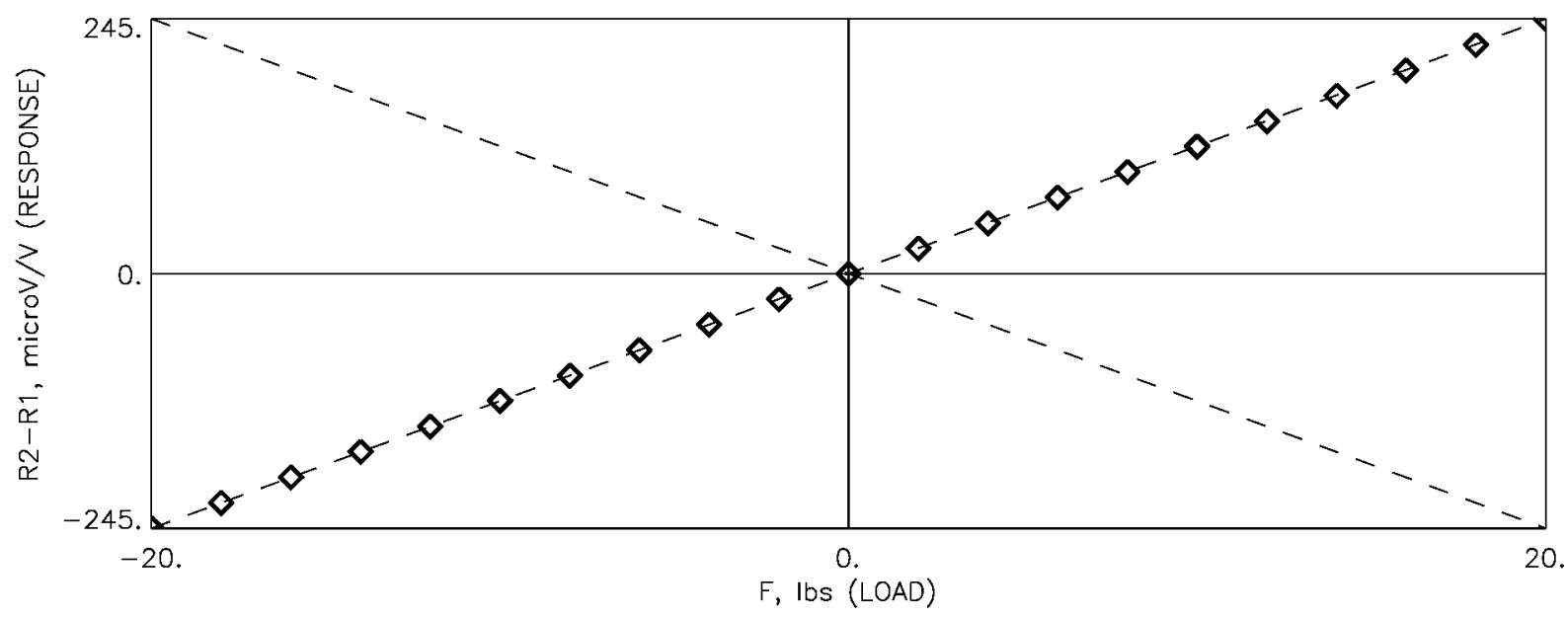

Fig. 5a Difference of gage outputs plotted versus the applied force at the balance moment center.

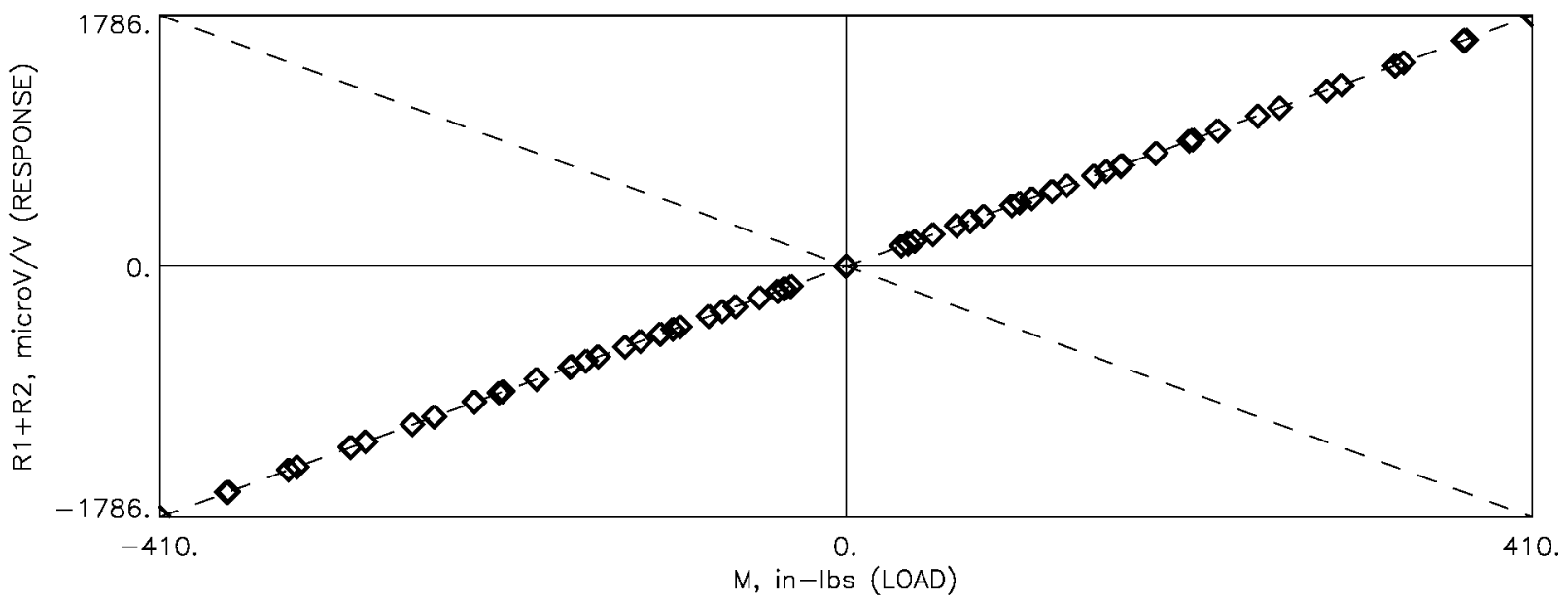

Fig. 5b Sum of gage outputs plotted versus the applied moment at the balance moment center. 
REGRESSION COEFFICIENT ESTIMATES AND STATISTICAL METRICS (R2-R1)

REGRESSION MODEL HIERARCHY CHARACTERISTICS - HIERARCHICAL

\begin{tabular}{|c|c|c|c|c|c|c|c|}
\hline $\begin{array}{l}\text { MATH TERM } \\
\text { INDEX }\end{array}$ & $\begin{array}{l}\text { MATH TERM } \\
\text { NAME }\end{array}$ & $\begin{array}{l}\text { COEFFICIENT } \\
\text { VALUE }\end{array}$ & $\begin{array}{l}\text { STANDARD } \\
\text { ERROR }\end{array}$ & $\begin{array}{c}\text { T-STATISTIC OF } \\
\text { COEFFICIENT }\end{array}$ & $\begin{array}{l}\text { P-VALUE OF } \\
\text { COEFFICIENT }\end{array}$ & $\begin{array}{c}\text { VIF } \\
\text { (PRIMARY) }\end{array}$ & $\begin{array}{c}\text { VIF } \\
\text { (ALTERNATE) }\end{array}$ \\
\hline 1 & INTERCEPT & -0.1051 & +0.0259 & -4.0605 & - & - & - \\
\hline 2 & $\mathbf{F}$ & +12.0427 & +0.0196 & +613.3586 & $<0.0001$ & +107.0591 & +107.0591 \\
\hline 3 & M & +0.0083 & +0.0010 & +8.4039 & $<0.0001$ & +107.0591 & +107.0591 \\
\hline 6 & $F * F$ & +0.0050 & +0.0134 & +0.3704 & +0.7117 & +6358.3359 & +6358.3359 \\
\hline 7 & $M \bullet M$ & $+5.6062 e-06$ & $+3.1574 e-05$ & +0.1776 & +0.8594 & +5290.8719 & +5290.8719 \\
\hline 10 & F.M & -0.0004 & +0.0013 & -0.2820 & +0.7784 & +22933.7747 & +22933.7747 \\
\hline
\end{tabular}

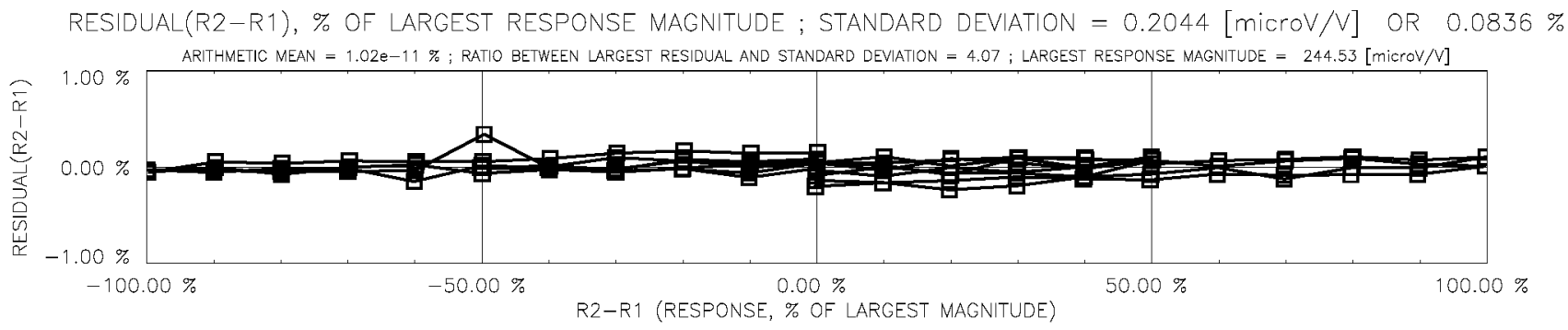

Fig. 6a Gage Output Difference: Least squares solution using a traditional quadratic as the regression model.

REGRESSION COEFFICIENT ESTIMATES AND STATISTICAL METRICS (R1+R2) REGRESSION MODEL HIERARCHY CHARACTERISTICS - HERARCHICAL

\begin{tabular}{|c|c|c|c|c|c|c|c|}
\hline $\begin{array}{l}\text { MATH TERM } \\
\text { INDEX }\end{array}$ & $\begin{array}{l}\text { MATH TERM } \\
\text { NAME }\end{array}$ & $\begin{array}{l}\text { COEFFICIENT } \\
\text { VALUE }\end{array}$ & $\begin{array}{l}\text { STANDARD } \\
\text { ERROR }\end{array}$ & $\begin{array}{l}\text { T-STATISTIC OF } \\
\text { COEFFICIENT }\end{array}$ & $\begin{array}{l}\text { P-VALUE OF } \\
\text { COEFFICIENT }\end{array}$ & $\begin{array}{c}\text { VIF } \\
\text { (PRIMARY) }\end{array}$ & $\begin{array}{c}\text { VIF } \\
\text { (ALTERNATE) }\end{array}$ \\
\hline 1 & INTERCEPT & +0.1407 & +0.0889 & +1.5827 & - & - & - \\
\hline 2 & $\mathbf{F}$ & +0.3110 & +0.0675 & +4.6085 & $<0.0001$ & +107.0591 & +107.0591 \\
\hline 3 & M & +4.3289 & +0.0034 & +1270.3971 & $<0.0001$ & +107.0591 & +107.0591 \\
\hline 6 & $F \bullet F$ & +0.1096 & +0.0459 & +2.3852 & +0.0186 & +6358.3359 & +6358.3359 \\
\hline 7 & $M \cdot M$ & +0.0002 & +0.0001 & +1.5687 & +0.1193 & +5290.8719 & +5290.8719 \\
\hline 10 & $F=M$ & -0.0091 & +0.0045 & -2.0298 & +0.0445 & +22933.7747 & +22933.7747 \\
\hline
\end{tabular}

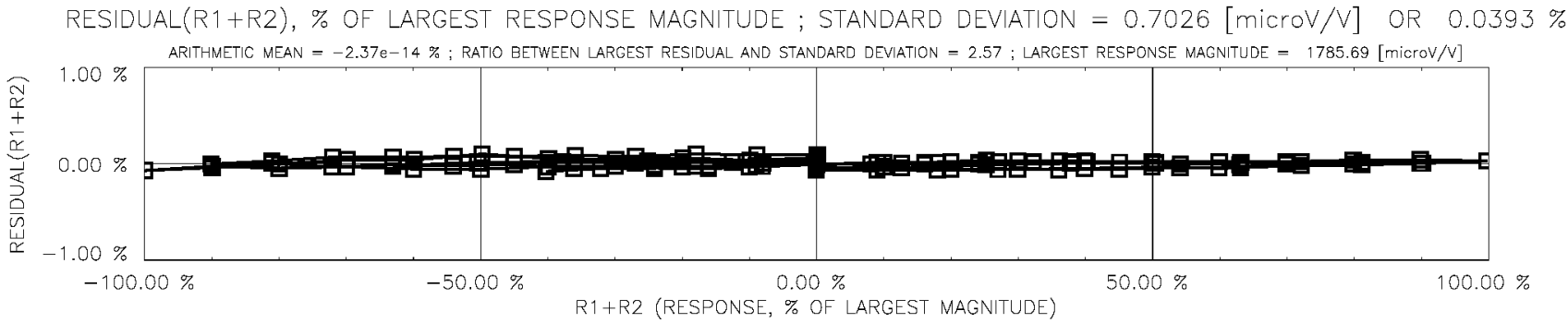

Fig. $6 \mathrm{~b}$ Gage Output Sum: Least squares solution using a traditional quadratic as the regression model.

American Institute of Aeronautics and Astronautics 
REGRESSION COEFFICIENT ESTIMATES AND STATISTICAL METRICS (R2-R1)

REGRESSION MODEL HIERARCHY CHARACTERISTICS = HIERARCHICAL

\begin{tabular}{|c|c|c|c|c|c|c|c|}
\hline $\begin{array}{l}\text { MATH TERM } \\
\text { INDEX }\end{array}$ & $\begin{array}{l}\text { MATH TERM } \\
\text { NAME }\end{array}$ & $\begin{array}{l}\text { COEFFICIENT } \\
\text { VALUE }\end{array}$ & $\begin{array}{l}\text { STANDARD } \\
\text { ERROR }\end{array}$ & $\begin{array}{c}\text { T-STATISTIC OF } \\
\text { COEFFICIENT }\end{array}$ & $\begin{array}{l}\text { P-VALUE OF } \\
\text { COEFFICIENT }\end{array}$ & $\begin{array}{c}\text { VIF } \\
\text { (PRIMARY) }\end{array}$ & $\begin{array}{c}\text { VIF } \\
\text { (ALTERNATE) }\end{array}$ \\
\hline 1 & INTERCEPT & -0.1116 & +0.0229 & -4.8676 & - & - & - \\
\hline 2 & $\mathrm{~F}$ & +12.2069 & +0.0024 & +5174.2210 & $<0.0001$ & +1.0000 & +1.0000 \\
\hline
\end{tabular}

RESIDUAL(R2-R1), \% OF LARGEST RESPONSE MAGNITUDE; STANDARD DEVIATION = $0.2583[\mathrm{microV} / \mathrm{V}] \quad$ OR $0.1056 \%$

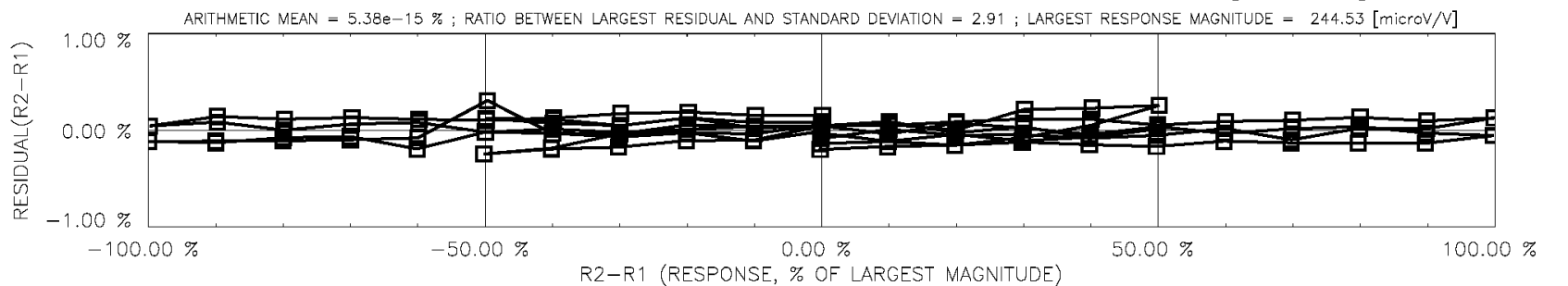

Fig. 7a Gage Output Difference: Least squares solution using BALFIT's optimized regression model.

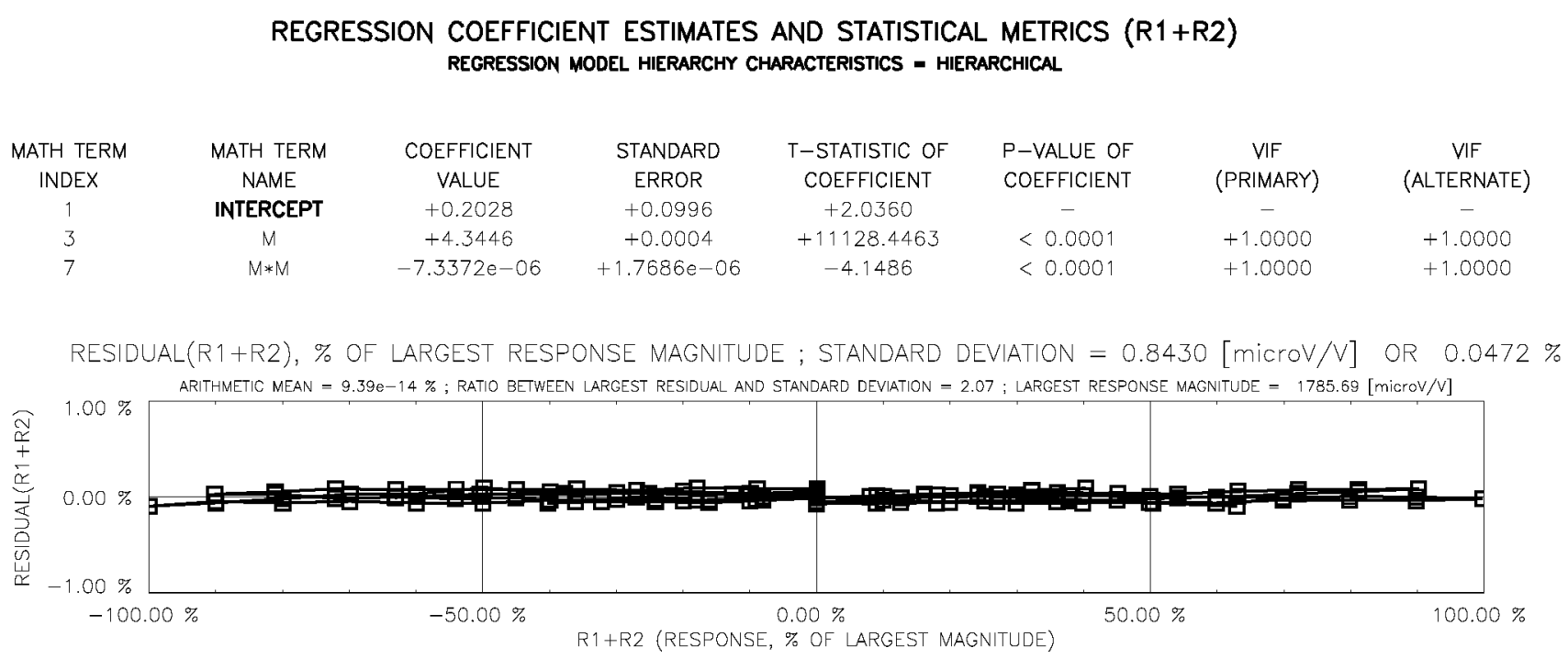

Fig. 7b Gage Output Sum: Least squares solution using BALFIT's optimized regression model. 


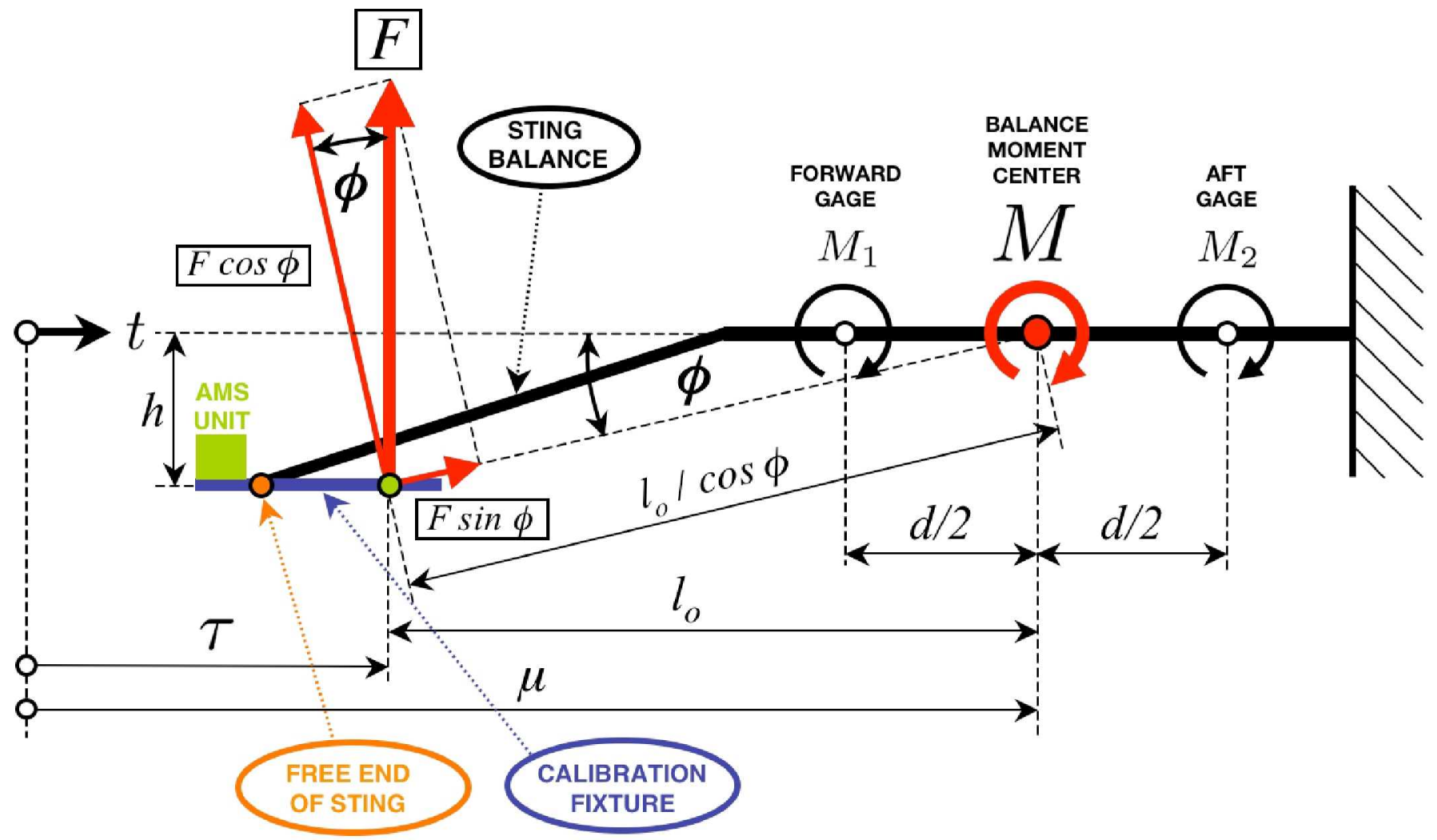

Fig. 8 Forces and moments acting on a sting balance during calibration.

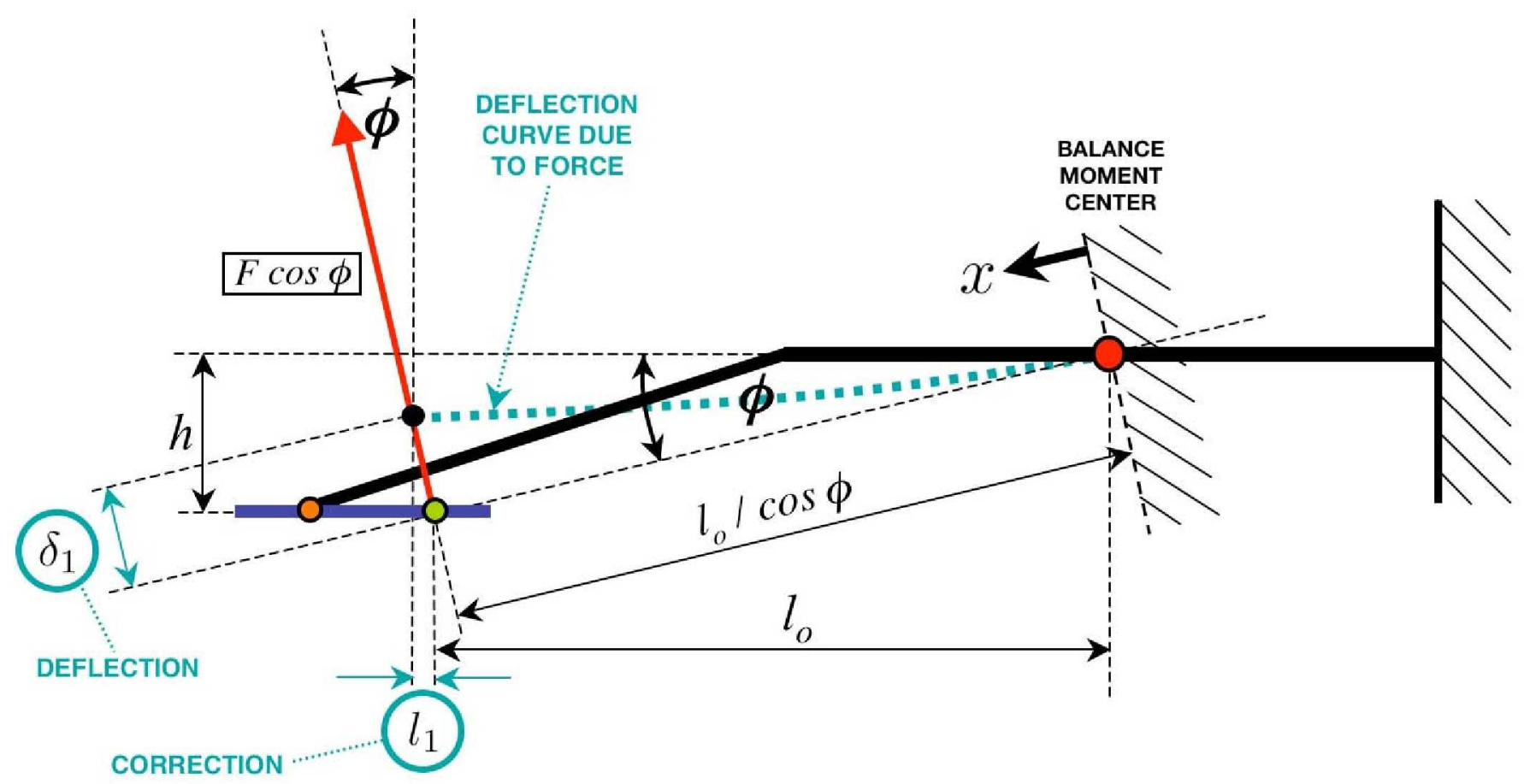

Fig. 9a Moment arm correction due to force induced beam deflection. 


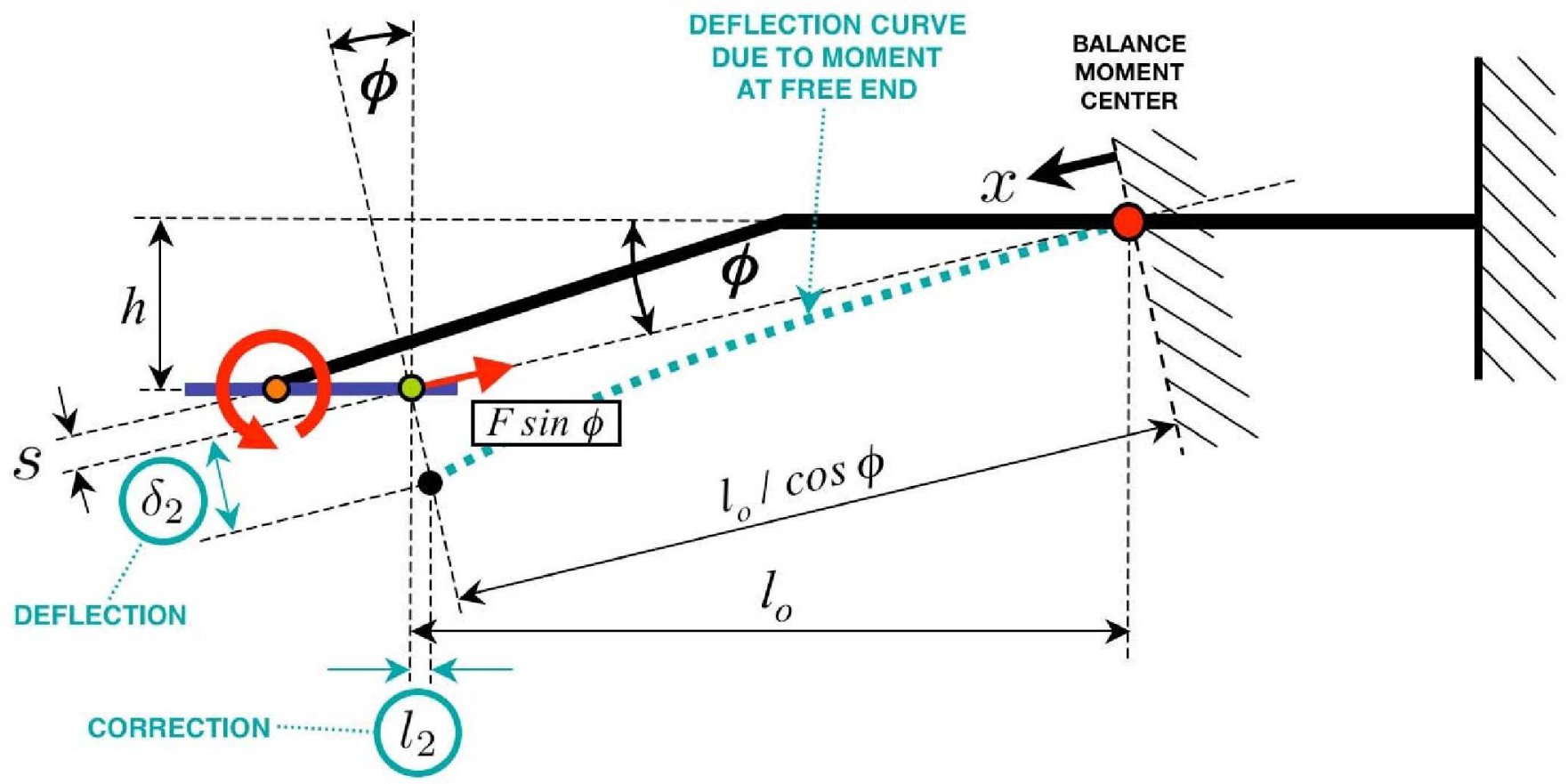

Fig. 9b Moment arm correction due to moment induced beam deflection.

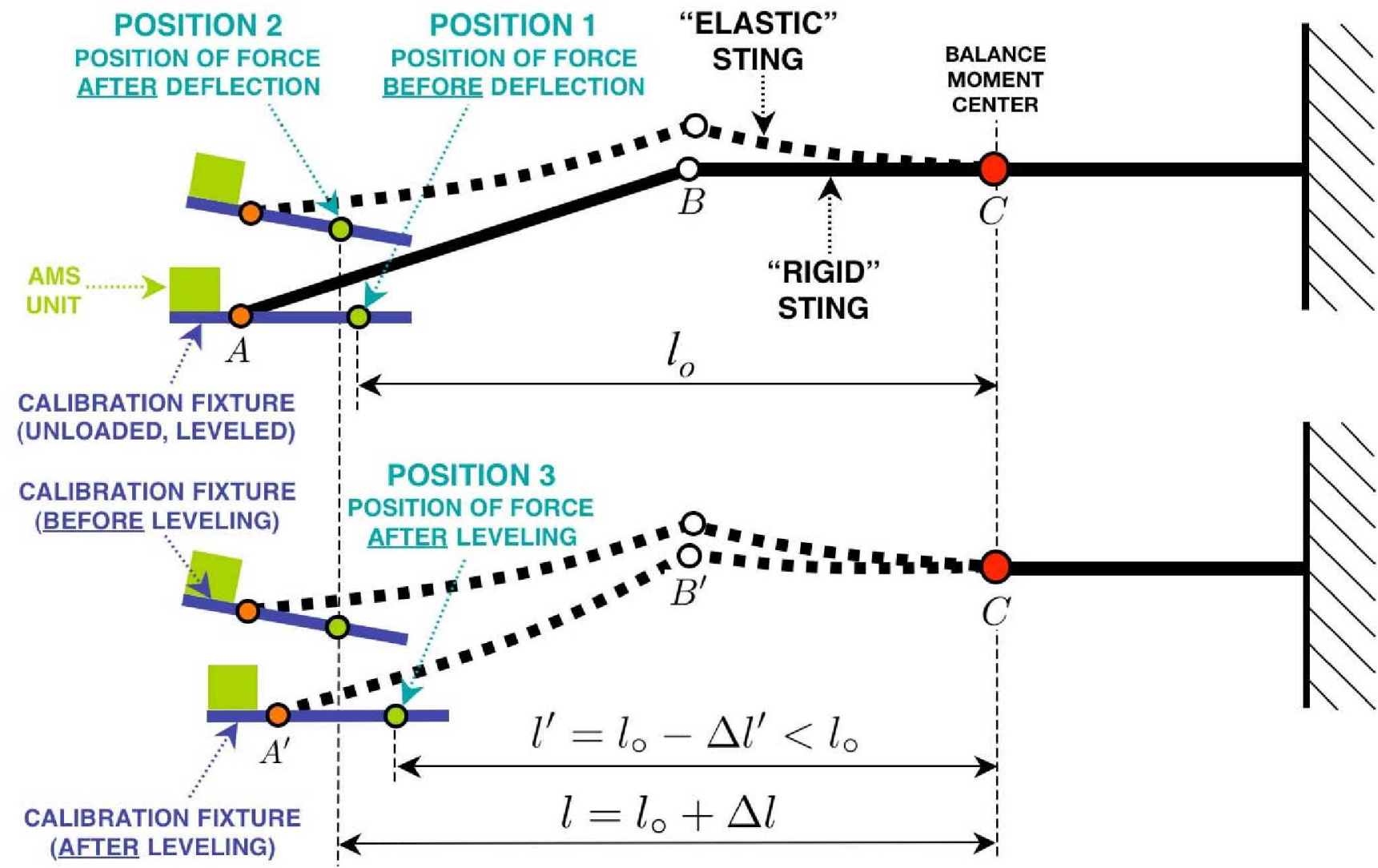

Fig. 10 Influence of (i) elastic sting deflection and (ii) calibration fixture leveling on the moment arm. 


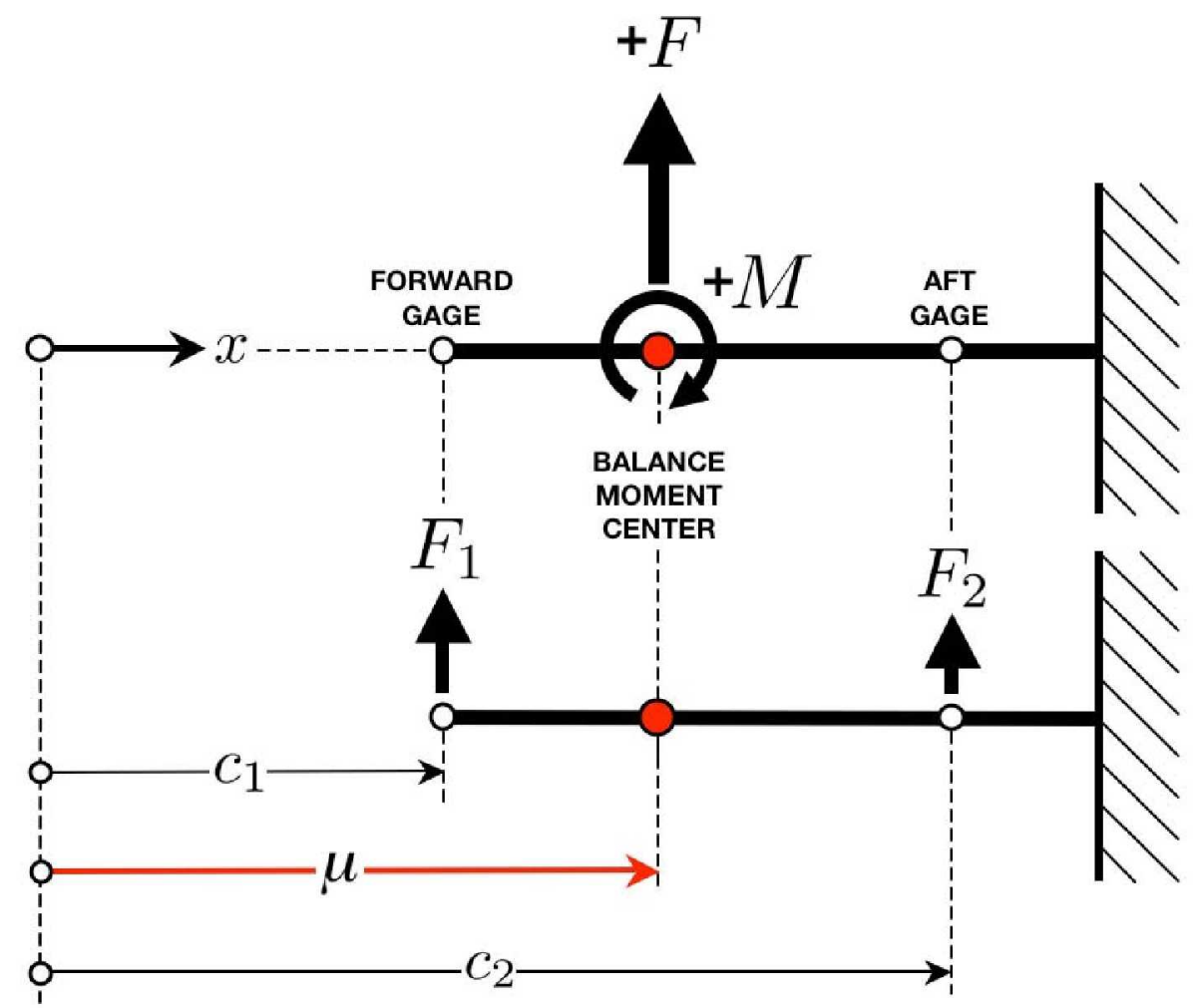

Fig. 11 Forces and moments acting at (i) the balance moment center and (ii) the gages of a strain-gage balance.

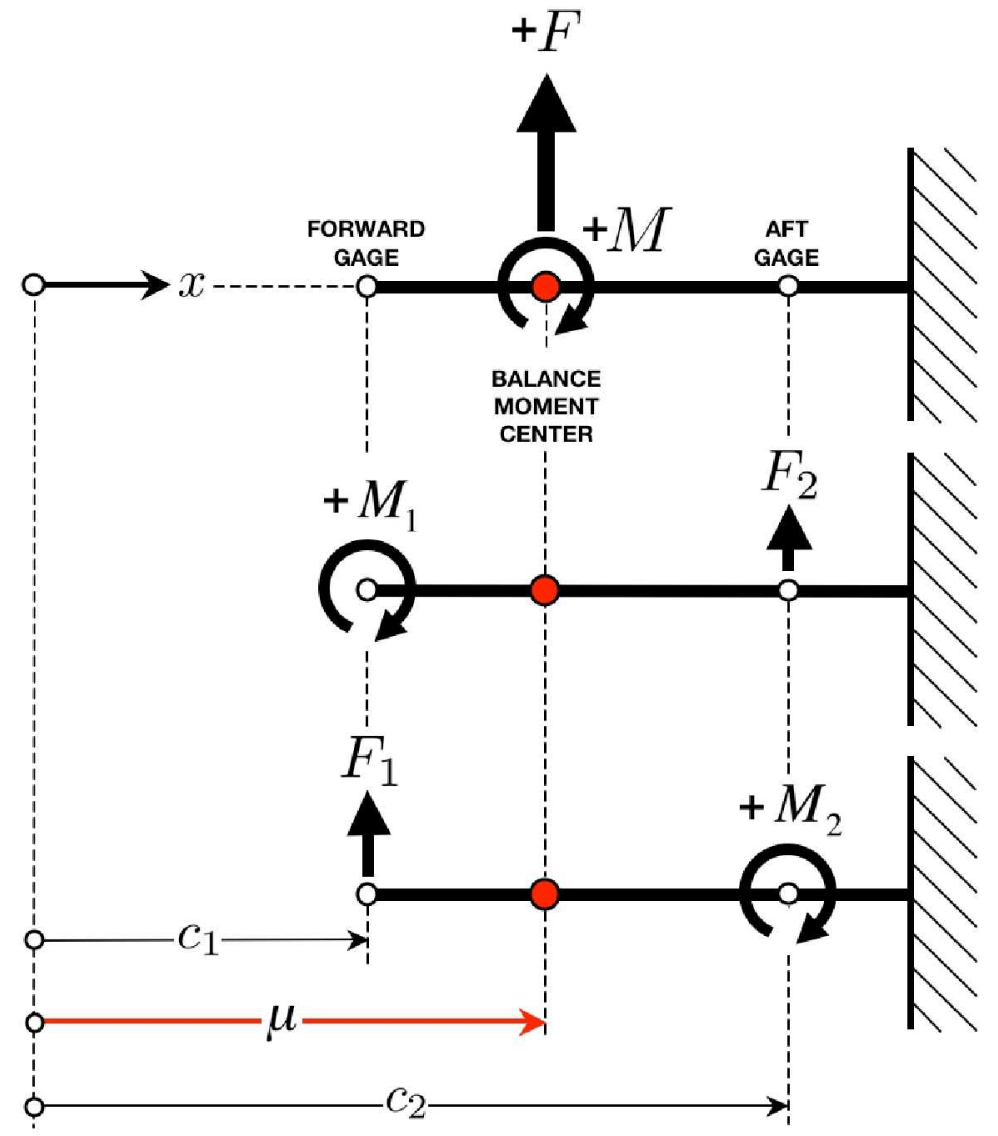

Fig. 12 Moments acting at the forward and aft gage of a strain-gage balance. 